\title{
Die deutsche Philosophie im Jahre 1912.')
}

Von Privatdozent Dr. Oscar Ewald.

Wenn wir in der Entwicklung der modernen Philosophie immer deutlicher die metaphysischen Probleme hervortreten sahen, so wird diese Wendung namentlich durch die Erscheinungen des letzten Jahres bestätigt. Sucht man nach einem vereinigenden, programmatischen Ausdrucke derselben, so wird man - namentlich im Hinblicke auf die deutsche Philosophie - sagen können, dass der Konflikt zwischen der reinen Erkenntnistheorie und der Metaphysik, der sich in den letzten Jahren verbreitete, offen ausgebrochen ist. Abgesehen von der Bereicherung, die das Denken hierdurch erfährt, besteht noch der Vorteil, dass die letzten grundlegenden Prinzipien auf beiden Seiten im Verlaufe der Diskussion mehr und mehr hervortreten müssen. Die reine Frkenntnistheorie, das ist jene Richtung, die den Begriff des Erkennens so weit spannt, dass er alle Fragen nach dem Sein der Dinge in sich absorbiert. Sie zerfällt wieder in zwei Gruppen: die phänomenalistische vider positivistische, die überhaupt nichts als real erkennt, was nicht Inhalt des Denkens ist; die logistische, die sich dem Seinsproblem gegenüber indifferent verhält, mit der Begründung, die Philosophie habe es bloss mit absoluten Normen und Werten, nicht aber mit absolnten Wirklichkeiten zu tun.

$\mathrm{Zu}$ wahrhaft klassischer Ausprägung ist der logistische Standpunkt im Neukantianismus der Marburger Schule gelangt, dessen namhafteste Vertreter Cohen, Natorp, Kinkel und Cassirer sind. Seine wesentlichen Tendenzen fasst Natorp in einem Aufsatze der Kantstudien (Festheft zu Hermann Cohens 70. Geburtstag), „Kant und die Narburger Schule" mit so glänzender Präzision zusammen, dass wir uns dieser Studie zur allgemeinen Orientierung bedienen könuen. Kant ist hier weniger in den $\mathrm{Re}$ -

1) Anm. d. Red.: Wir verweisen wiederum auf unsere, dem ersten, Bd. XII veröffentlichten, Jahresbericht beigefïgte Notiz.

Kantstudien XVIII. 
sultaten als in der Methode des Denkens vorbildlich. Die Methode aber weist auf Plato zurück, der das. Wesen des Denkens zum ersten Male nicht als starre Fixierung, sondern als unendliche Bewegung verstanden hat. Unendliche Bewegung ist das Denken auch für Kant. Es gibt nichts, das nicht von diesem Prozess der Bewegung ergriffen würde, keine ruhenden Gegenstände, keine Dinge an sich, an die das Denken gleichsan von aussen heranträte, um ihm seine Formen aufzuprägen. Solcher Realismus steht in Widerspruche mit dem Leitmotiv der transzendentalen Methode, deren Sinn es ja ist, dass alle Gegenständlichkeit lediglich eine bestimmte Struktur des Denkens ist. Das Denken selbst in seiner kategorialen Gesetzmässigkeit erzeugt den logischen und wissenschaftlichen Grundbegriff des Dinges, der vom naiven Berrusstsein fälschlich jeuseits aller Denkbarkeit hinausverlegt wird. Ein absolut Denkfremdes kann es uicht geben; das verbietet die von Kant vollzogene "kopernikanische" Unkehrung des Standpunktes, dergemäss das Sein im Denken seine tiefste Begründung findet. Die Kategorien dürfen aber auch nicht als fixe Schemen und Schablonen des Geistes betrachtet werden, ein Irrtum, von den Kant nicht völlig frei ist, sie dürfen nicht von eiven unbeweglichen Punkte aus dirigiert, sondern muissen in ihrer wechselseitigen funktionalen Beziehung und Bedingtheit erfasst werden, sie müssen sich in den sie erzeugenden lebendigen Denkprozess selbst auflösen. Diese Methode der Korrelation an Stelle einer geradlinigen Deduktion hat Natorp in Anschlusse an Cohens "Logik der reinen Erkenntnis" in scinen "Logischen Grundlagen" auch selbständig in Angriff genommen. Unverkennbar ist ihre Abhängigkeit von der Hegelschen Dialektik, aber auch die Verschiedenheit beider Denkweisen. Letztere liegt nicht allein wie Natorp vermeint - in der absolutistischen Tendenz Hegels, in seinem Anspruche, die Totalität des.Denkens erschöpft zu haben, sondern vor allem darin, dass der Panlogismus der Identitätsphilosophie Metaphysik ist, während der neukantische Panlogismus auch in seiner äussersten Erweiterung Logik bleibt. Für Hegel ist der absolute Geist nicht bloss Bestimmung, sondern Erschaffung des Seins durch das Denken. Indem das Logische dergestalt nicht mehr die Formen der Dinge umschreibt, sich von aussen wie ein Rahmen um sie spannt, indem es in ibr Innerstes eindringt, wird es zur weltschöpferischen Gewalt. Oder wie wir auch sagen können: im Neukantianismus ist es bloss ein Wert, 
im Hegelianismus ist es auch eine Macht. Mag es paradox klingen, Hegel hat ein tiefdringendes Verständnis für den irrationalen Charakter des Seins; soll das Denken seiner Herr werden, so muss es etwas von seinem Wesen in sich aufnehmen, was sich in der Dialektik kundgibt, die ja eine merkwürdige Durchdringung von Rationalität nnd Irrationalität ist. Es darf sich ferner nicht darauf beschränken, sinnvolle Bedeutung zu sein, es muss ein schöpferisches $\mathrm{Tun}$ werden, es muss sich mit der eingeborenen Dynamik des Seins sättigen. Dem Neukantianisnus kann es freilich nicht zum Vorwurf gemacht werden, dass er das Sejn einseitig logisiert hat; solches verbietet ja schon die Tradition Kants, die nirgends so eindringlich baftet wie an der Widerlegung des ontologischen Gottesbeweises, an dem hier ausgesprochenen Verbote, die Denkbarkeit mit dem Sein, den Begriff mit dem Realen zu verwechseln. Vielmehr ist das Sein für den Neukantianismus der Ausdruck einer Relation, der Exponent zweier wesensdifferenter Glieder: der Phänomene einerseits, der Kategorien andererseits. Weder durch die sinnlichen Erscheinungen wird das Sein begründet - es ist ja in ihnen kein Kriterium der Realität enthalten, ebensowenig wie in blossen Träumen oder Phantomen - noch aucb in den Kategorien, die ja an sich leer sind und, um mit der Realität überhaupt in Berührung zu kommen, der sinnlichen Erfüllung bedürfen. Sondern die fortschreitende $\mathrm{Be}$ stimmung des Sinnlichen durch das Z̈bersinnliche, der Phänomene durch die Kategorien, die, wie Natorp hervorhebt, eine unendliche, niemals zum Abschluss kommende ist, konstituiert das Sein, das sich so dennoch in einen permanenten logischen Prozess auflöst. Diese Auffassung ist insofern ein Phänomenalismus, als sie, auf Metaphysik prinzipiell Verzicht leistend, den Umkreis des Bewusstseinsinhaltes nicht überschreiten will. Dies ist ihm auch mit dem Positivismus gemeinsam, von dessen landläufigen Formen er sich freilich durch seine logistische Teudenz unterscheidet. Ein Transzendieren des Bewusstseins in der Richtung anf das Ding an sich verbietet der Neukantianismus schon aus dem Grunde, weil ihm Sabjekt und Objekt und die im Erkenntnisakt sich manifestierende Beziehung beider gar keine unmittelbar gegebenen Realitäten, sondern logische Positionen sind. Das Denken setzt erst den Begriff der Gegenständlichkeit, mag sich dieser weiter zum Objekt oder Subjekt besoudern. Es setzt ferner die Korrelation zwischen beiden; und ebenso konstruiert es als Grenzbegriff den 
dieser Korrelation enthobeuen Begriff eines A bंsoluten, eines „Ding an sich". Wohin wir also immer mit unserer Betrachtung dringen mögen, stets stehen wir schon vor einem logisch Geformten; nicht erst Subjekt und Objekt, schon der einfache, scheinbar unteilbare Empfindnngsakt erweist sich genauerer Analyse als solches. Gleichwohl ist dieser Standpunkt kein panlogistischer: denn er nuss anerkennen, dass es ein Letztes, wenn auch völlig Dunkles und Bestimmungsloses gibt, das eben immer und überall in logische Kategorien gestellt wird, von logischen Bestimmungen gleichsam durchwachsen ist. Ein solches Substrat ist auch dem Neukantianismus unentbehrlich, wenngleich es niemals in nackter Unmittelbarkeit gegeben ist. Es geht aus dem Bisherigen hervor, dass er es auch anerkennt, dass für ihn der logische Prozess nicht ohne Rest aufgeht und eben deswegen ein unendlicher ist. Damit ist aber auch schon eine Grenze des Logischen im Gegebenen gesetzt, ein prinzipieller Gegensatz, der nicht aufgehoben werden kann. Ist der Hegelsche Panlogismus, wie wir selien werden, monistisch, so bleibt auch der extreme Neukantianismus in den Dualismus gebanut. Das nuss aber auch einen entscheidenden Einfluss anf die Fassung und Begründung des Seinsbegriffes äussern. Die kühnste, originellste Leistung des Neukantianismus nämlich, in der er die Konsequenz des transzendentalen Motivs ziehen will, ist die Auflösung des Seins in ein Gelten, der Existenz in pure Essenz, der Realität in ideale Werte. Der Vorgang ist hier der folgende: da das Sein ein Verbältnis zwischen dem Irrationalen und dern Rationalen bezeichnet, ist es nicht Substanz, sondern Fuuktion, wie das ausser von Cohen und Natorp namentlich von Cassirer erläutert wird. Es ist die Bewegung der fortschreitenden Ratiovalisierung, welche an keinem Punlt zur Ruhe gelangt. Die Form dieser Bewegung ist das Urteil, dessen Wesensart die Durchdringung und Korrelation des Besonderen und Allgemeinen enthält. Das Urteil aber ist wie jede logische Funktion in idealen, zeitlos giltigen Werten begründet. Sonach ist das Sein selber im Gelten verankert, ja es gibt kein anderes Kriterium des Seienden als das Giltige der Existenz, als die Essenz. Namentlich Rickert, der seine Theorien abseits vom strengen Kantianismus entwickelt hat, zieht diese Konsequeuz; und man kann sagen, dass sie sich in einem weiten Umkreise der modernen Philosophie bemächtigt hat. Erst in jüngster Zeit ist in wachsenden Masse eine Opposition gegen diesen extremen Standpunkt wahrzunehmen, deren 
zum Teil schon im vergangenen Jahresbericht Erwähnung geschah. So zwingend nämlich die Logik scheint, die vom Sein zum Gelten als der höheren Instanz führt, sieht man näher zu, so findet man, dass sie nicht auf dem Fundamente ruht, welches das kantische System trägt; dass im Gegenteile ein Grundpfeiler derselben durch sie ins Wanken gerät. Der Kritizismus nämlich ist ein absoluter Dualismus und zwar ein solcher zwischen der Rationalität und dem Irrationalen. Als irrational wird das Faktum der Existenz gesetzt. Das Merkmal des Logischen ist ja Daseinsfreiheit; und umgekehrt ist das Sein dadurch charakterisiert, dass es als solches sich nicht logisieren lässt. Dies bedingt zum Beispiel den Unterschied zwischen den mathematischen und dynamischen Kategorien hinsichtlich ihrer Evidenz. Auf diesen Dualismus fussend, zertrümmert Kant auch das Prinzip der alten und dogmatischen Ontologie, das sich namentlich im Gottesbegriff festgesetzt hatte: als ob die Existenz als logisches Merkmal aus der Essenz eines Begriffes deduziert werden könnte. Sicherlich weicht das Verfahren des modernen Logismus von demjenigen der Ontologie ab, schon deswegen, weil die Stellung zur Metaphysik eine andere geworden ist. Aber im letzten Prinzip herrscht noch immer eine Gemeinschaft: in der Verkennung der absoluten Distanz, die zwischen dem Dasein und der Begrifflichkeit waltet. Auch der moderne Logisnus ordnet die Existenz dem Denken ein und unter, ob er diese Hineinnahme als eine vollzogene Tatsache oder als einen uuendlichen Prozess betrachte. Das entscheidet auch über sein Verhältnis zum Ding an sich, überhaupt zum Problem der Transzendenz. Es ist sehr wesentlich, dass er das Ding an sich völlig zu rationalisieren trachtet, es zur Vernunftidee, ja zur Grundfunktion des vernünftigen Intellektes stempeln möchte, während Kant es ganz dentlich als denkfremden Faktor gesetzt, auf den die Empfindung als ihren Seinsgrund hinweist. Dass etwas existiert, ist Voraussetzung der angewandten Logik: als dasjenige, worauf sie Anwendung findet; es kann daher nicht aus ihr selber abgeleitet und begriffen werden. Es scheint, dass eine Theorie, die solches verkennt, das Merkmal der Gegenständlichkeit mit dem der Existenz verwechselt. Gegenständlichkeit, mag sie sich jetzt als subjektives oder objektives Sein qualifizieren, ist zweifellos ein durch und durch Kategoriales. Sie ist nicht Existenz schlechthin, sondern schon logisch geordnete und logisch durchdrungene und bewältigte Existenz. Die Tatsache, dass 
überhaupt etwas existiert, bleibt für den kritischen Philosophen eine absolute, $\nabla 0 m$ logischen Erkenneu uuüberschreitbare Grenze. Und diese Grenze vermochten auch die nachkantischen Denker, die Identitätsphilosophen, nicht dadurch zu überschreiten, dass sie das Sein im logischen Gelten aufgehen liessen, sondern umgekehrt - wie dies am Beispiele Hegels angedeutet wurde - dass sie ein Seiendes suchten, dass in sich selbst den absoluten, idealen Charakter logischer Werte präsentierte. Mit einem Worte: durch den Übergang von der Logik zur Metaphysik.

Aber auch wenn man unter Existenz bloss gegenständliche Existenz versteht, ist die Seinsfrage nicht definitiv ausgeschaltet. Es bandelt sich dann bloss um eine terminologische Verschiebung. Es muss dann für die logisch noch nicht bewältigte Masse der Phänomene, die zugleich Material des Logischen. ist, ein eigener Seinsbegriff konstituiert werden, der Begriff einer Realität, welche, wenn sie im Vergleich mit der gegenständlichen auch nicht als vollwertig betrachtet wird, nichtsdestoweniger eine spezifische, unentbelhrliche Bedentung besitzt. Der Standpunkt des Logismus setzt dann den eines radikalen Phänomenalismus voraus. Das unmittelbar Gegebene sind Erscheinungen und zwar schlechtweg unbestimmte Erscheinungen. Indem sich das logische Denken ihrer bemächtigt, ihnen seine Formen aufprägt, entsteht erst die Gliederung in Subjekt und Objekt sowie alle sonstige Einstellung in Reihen und Systeme, als deren Resultat sich ein geordneter Weltbegriff ergibt. Der Inhalt aber kann letzten Endes nicht von der Logik hervorgebracht werden, er muss ihr - mag man dies Verhältnis noch so sehr umzudeuten streben - irgendwie gegeben sein. Man kann ihn ja auf ein Minimum der Bestimmtheit und das heisst zugleich des Wertes und des Seins beschränken: völlig eliminieren lässt er sich nicht. Und damit ist nicht allein das Irrationale eingeräumt, sondern zugleich in engste Verbindung mit der. Tatsache des Seins gebracht. Dass die Grenzen des Logismus in ihm selber gelegen sind, ist bereits in mehrfacher Weise, so vornehmlich von Lask in seiner "Logik der Philosophie“ zum Ausdrucke gebracht worden, die durch diese Feststellung den Rahmen des Neukantianismus sprengt. Das Logische umkleidet sein Material mit begrifflicher Bestimmtheit, aber es dringt uicht in dasselbe ein, es in ein Begriffliches zu verwandeln. Dazu kommit noch eine Erwägung, die gerade durch den Gedankengang des logischen Idealismus bedingt erscheint. Indem das ausser- 
logische Material begrifflich gemeistert wird, verbindet sich mit diesem Prozess schou der Hinweis auf einen Schöpfer oder Träger der begrifflichen Funktionen. Will man denn wirklich ein Denken annehmen, das von jedem denkenden Bewusstsein abgelöst, sich selbst überlassen, in gleichgewichtsloser Schwebe bleib!? Der Wert, die Geltungssphäre des Gedachten mag autonomen, von jedem Bewusstsein unabhängigen Bestand haben. Im Werte des Gedachten erschöpft sich aber nicht die Tatsache des Denkens; diese ist immer an ein denkendes Wesen gebunden. Und so zeigt es sich, dass die Gliederung in Subjekt und Objekt, die der Logist erst aus dem Denken hervorgehen lassen möchte, in Wahrheit vom Denken bereits vorausgesetzt wird. Dies ist ja der Sinu des von Descartes entdeckten Grundsatzes, der am Eingange' der modernen Philosophie steht. Auch wo der Wert des Gedachten noch völlig strittig ist, erscheint die Realität des Denkens und damit des denkenden Ich gesichert. Der Logist wird dieser Konsequenz entgehen, indem er das reine Denken als einen gänzlich unpersönlicheu Prozess betrachtet, der das empirische Subjekt nicht voraussetze, soudern es erst fortschreitend erzeuge. Dann aber muss als Träger des Denkens ein überpersönliches, metaphysisches Bewusstsein gefordert werden. Und wirklich sehen sich viele Logisten zu dieser Konsequenz getrieben, der sie freilich in dem Begriff des „Bewusstseins überhaupt“ einen sehr unsicheren und schwankenden Ausdruck geben. Schon in meinen früheren Jahresberichten habe ich gezeigt, dass als Träger eines wirklichen Denkprozesses bloss ein reales Bewusstsein bestehen kanu, in unserem Falle mithin ein reales Allbewusstsein wie der Hegelsche Weltgeist. Zweifellos ist aber hier das Mass der metaphysischen Behaftung ein viel grösseres, als dort, wo vom Denken bloss auf ein empirisches Ich zurïckgegangen wird.

Uńser Ergebnis bleibt also, dass alles Denken eine Realität voraussetzt, deren wir unmittelbar innewerden, sei es auf dem Wege der Empfindung, des Willens oder der Intuition. Diese Betrachtungen berühren sich in mancher Beziehung mit den Argumenten, die Frischeisen-Köhler in seinem Vortrag "Das Realitätsproblem: (veröffentlicht von der Kantgesellschaft, Verlag von Reuther \& Reichard, Berlin, S. 98) gegen den logistischen Standpunkt erhebt. Er weist die Auffassung zurück, dass Realität eine logische Kategorie ist, indem er zunächst auf die selbständige Beziehung der Empfindung und sodaun des IVillensaktes zum Sein 
sich beruft. Der Begriff der Aussenwelt wird nicht auf intellektualistischem sondern auf voluntaristischem Wege gewonnen. Indem wir mit unserem tätigen Wollen auf einen Widerstand stossen, werden wir fremder Existenz unmittelbar inne. Das Bewusstsein vom Objekt. wird sonach in eine vorlogische Sphäre verlegt. Dieșer Rekurs auf den Willen ist die Fortführung eines zumal von Dilthey mit Energie dargestellten Motirs, das freilich auf Maine de Biran zurückreicht. Er hat auch eiue unverkennbare Ähnlichkeit mit dem modernen Intuitionismus. Dies Prinzip einer unmittelbaren Erkenntnis führt hier freilich bloss zu einem empirischen, nicht zu einem absoluten Realismus. Die Realität der Gegenstände ist vom empfindenden und denkenden Bewusstsein unabhängig, nicht aber von allen Bestimmungen des Bewusstseins überhaupt. Es liegt ja schon in Begriff des Wollens und Handelns, dass der erfahrene Widerstand lediglich in Korrelation zum Ich als sein Gegenglied erfahren wird. Der absolute Realismus dagegen ist insoferne dogmatisch, als er Merkmale, die innerhalb des Bewusstseins gewonnen werden, auf eine völlig transzendente Sphäre überträgt. Ausführlicher hat der Verfasser diesen Standpunkt in der Schrift "Wissenschaft und Wirklichkeit" entwickelt (Verlag Teubner, Leipzig, VIII u. $478 \mathrm{~S}$.), die sich in drei Hauptteile gliedert, derèn erster die Unzulänglichkeit des transzendentalen Idealismus zu beweisen hat; deren zweiter in einer Phänomenologie des Realitätsbewusstseius die logisch unauflösbare Eigenart der Wirklichkeit darstellen soll. Auch hier wird der von Dilthey hervorgehobene Zusammenhang mit dem Impuls des Wollens betont und so der Anteil des unmittelbaren Lebens am Aufbau des Realitätsbegriffes geltend gemacht. Ein grossangelegter Versuch, den Realismus zu rechtfertigen und zu begründen, ist Oswald Külpes Werk „Die Realisierung“ (Verlag von Hirzel, Leipzig, $\dot{X}$ u. 257 S.), dessen erster Band vorliegt. Schon der Titel bringt die Tendenz zum Ausdrucke: es handelt sich darum, die not.wendige Beziehung des Erkennens auf eine selbständige, in sich beruhende Realität aufzuzeigen. Külpe zieht den Kreis des Problems möglichst weit; nicht allein in den Naturwissenschaften, im Begriff der Aussenwelt, stehen wir vor der Notwendigkeit solch einer Beziehung und Setzung, sondern auch in der Psychologie und in den Geisteswissenschaften überhaupt. Hier wie dort weist das Denken über sich hinaus, gewinnt es seinen Sinn und seine Richtungslinien erst von der Annahme einer in 
ihm unauflösbaren Realität. Die bisherigen Versuche, den Bann des Idealismus zu überwinden, zum Beispiel Krafts im letzten Jahresberichte erwähnte Schrift „Erkenntnisbegriff und Weltbegriff“ hatten sich etwas einseitig am Aussenweltproblem orientiert; hier dagegen ist die Gesamtsphäre des Seins in die Problemstellung einbezogen. Und so gilt es auch in der Sicherstellung des realistischen Motivs gegen äussere Angriffe eine möglichst breite Basis der Defensive zu gewinnen. Es muss Külpe als ein besonderes Verdienst angerechnet werden, dass er vielleicht zum ersten Male völlig deutlich zwei verschiedene Arten des Idealismus auseinanderbält: den Konszientialismus oder Bewusstseinsidealismus und den transzendentalen, logischen Idealismus. Bisher wurden diese beiden Arten, die ja auch unzweifelhaft bestimmte Berührungspunkte haben, in der Regel zusammengeworfen. Und dennoch hat die Analyse des Neukantianismus uns gezeigt, dass wenigstens die dusdrucksform des logischen und des Bewusstseinsidealismus eine wesentlich verschiedene ist. Der Bewusstseinsidealismus, der überall auf Hume oder eigentlich auf Berkeley zurückweist, ist eine Seinsphilosophie. Bloss vereinfacht und vereinheitlicht er das Seiende, indem er es überall auf geistiges Sein zuräckführt. Er legt der gesamten Realität eine bestimmte Form derselben, die spirituelle zugrunde. Der logische Idealismus hingegen ist eine Geltungsphilosophie; er löst das Reale in ein Ideales, die Existenz in eine Essenz, das Seiende in ein Geltendes auf. Külpes Argumente gegen den Konszientialismus bewegen sich in der Richtung realistischer Gedankengänge. Indem wir mit unserem Denken ein Wirkliches aufbauen, gehen wir stets über die unmittelbaren fliessenden Inhalte des Bewusstseins hipaus. Mit ausserordentlicher Gründlichkeit werden alle Argumente des Bewusstseinsidealismus gewürdigt und widerlegt: Die Berufung auf die angebliche Evidenz der inneren Wahrnehmung, die vermeintlichen Vidersprüche im Begriff der Transzendenz, das tatsächliche Gegebensein aller Dinge im Bewusstsein, die Zweckmässigkeit eines Verzichtes auf Realisierung etc.

Die Unmittelbarkeit des Bewusstseins transzendieren wir indessen nicht, um uns bloss zu idealen logischen Normen und Werten zu erheben, vielmelir um eine metuphysische Wirklichkeit zu setzen. Hier fällt das Hauptgewicht auf den prinzipiellen Unterschied, den Külpe zwischen dem Verfahren der Mathematik und dem der Realwissenschaften feststellt. In der Mathematik 
crzeugt dus Denken fortschreitend seine eigenen Gegenstände, ohne Rücksicht darauf, ob diese irgend einer Realität angehören oder nicht. In den Realwissenschaften hingegeu ist das Denken durch die Gegenstände gebunden, die ihm von sich aus die Richtung vorscbreiben. Der idealistische Einwand, anch hicr seien die Gegenstände kein fertig Gegebenes, ist insoferne berechtigt, als' das Denken nirgends ein blosses Abbilden, vielmehr ein Konstruieren und Bestimmen ist. Aber diese Konstruktion und Bestimmung ist hier keine freie, von bloss idealen Gesetzen, sondern eine von dem tatsächlichen Material beherrschte. Ähnlich wie Frischeisen-Köhler weist Külpe auf die "empirische Behaftung" des Denkens in den Realwissenschaften hin. Ond wenn es auch wahr ist, dass wir hier nirgends zu dem reinen Stoffe als einer absoluten Grenze des Logischen gelangen, so ist es doch andererseits ebeuso wahr, dass wir hier nirgends wie in der Mathematik ein rein Logisches, gelöst von der Beziehung auf den stofflichen Vorwurf haben. Die kategorialen Denksetzungen stellen sich nicht bloss immer an einem irrationalen Materiale dar, sie scheinen auch Schritt, für Schritt durch dasselbe bestimmt. Külpe warnt hier ebenso vor den Extrem des naiven Realismus wie des absoluten Logismus: der kritische Realismus hat zwischen beiden die Mitte zu halten. Das Erkennen ist weder eiu Abbilden noch ein Schaffen; es ist ein Erfassen der nicht gegebenen, aber durch (xegebenes sich offenbarenden Realitäten. Die transzendentale Methode, wie sie durch Kant begründet wurde, hebt die reale Existenz der Dinge nicht anf, so sehr sie ihre Erfassung und Bearbeitung auch unter aprioristische ideale Bedingungen stellt.

Zugunsten der realististischen Ansicht suchen auch zwei Schriften von Alfons Bilharz das Erkenntnisproblen zn lösen: "Descartes, Hume und Kant" (S. 78) und „Philosophie als Universalwissenschaft" (S. 127, Verlag. Bergmann, Wiesbaden). Der ontologische „Enantialsatz“, der, die beiden gegensätzlichen Bestimmungen Denkeu und Seiu im Ich verbindet, garantiert zugleich die Begreuztheit des subjektiven Seius durch ein seiendes Objekt. Diese Begrenztheit, die der Verfasser als metaphysische Form bezeichnet, soll die idealistischen Prämissen in realistische Konsequenzen verdichten.

Ich kehre nunmehr zu der Darstellung zurück, die der logische Idealismus im Festhefte der Kantstudien erfährt. . Zu einem ähulichen Ergebnis wie Natorp gelangt Cassirer in seinem Auf-. 
satze „Hermann Cohen und die Erneuerung der Kantischen Philosophie ". Hier ist, der persönlichen Richtung Cassirers entsprechend, freilich weniger das panlogistische Element, als der Zusammenhang mit den konkreten Wissenschaften, insbesondere mit der Mathematik und der Physik betont. Die Bedeutung dieses Zusammenhanges ist es namentlich, den logischen Idealismus vor jedem Rückfall in den psychologischen zu sichern. Bewusstsein ist das Inventar wissenschaftlicher Begriffe, nicht aber subjektiver Vorstellungen. Aber auch die Orientierung an den Grundbegriffen der mathematischen Physik bedeutet keine Orientierung an starren Gegebenheiten und demnach auch kein Verstricktsein des philosophischen Denkens in die historische Relativität des wissenschaftlichen Prozesses. „Die Gegebenheit, die der Philosoph in der mathematischen Naturwissenschaft anerkennt, bedeutet eben zuletzt lediglich die Gegebenheit des Problems. In ihrer tatsächlichen Form sucht und erkennt er eine ideale Form, die er heraushebt, um sie wiederum den wechselnden historischen Gestaltungen als Massstab gegenüberzustellen. Wenn hierin ein scheinbarer Zirkel liegt, so ist es doch ein Zirkel, der unvermeidlich ist, weil er auf jener Wechselbewegung von Idee zu Erfahrung beruht, durch die - nach dem Goetheschen Wort - die sittliche und wissenschaftliche Welt regiert wird." Damit ist jedenfalls zugegeben, dass der philosophische IVirklichkeitsbegriff ein antonomer ist, dessen Rechtfertigung nicht in dem methodischen Verfahren anderer Forschungsgebiete gewonnen werden kanu. Ins Detail gehen die Aufsätze Görlands "Hermann Cohens systematische Arbeit im Dienste des kritischen Idealismus" und Kinkels „Das Urteil des Ursprungs". Görland charakterisiert deu Ü̈bergang Cohens von der strengen Kantinterpretation zum freiereu Auslau seines Systems der Philosophie. Massgebend hierfür ist Cohens Auffassung von der Infinitesimalmethode als dem erzeugenden Element des Realitätsbegriffes. Realität nämlich muss von Wirklichkeit, Sein von Dasein unterschieden werden. Dasein, Wirklichkeit, eignet der Empfindung, die als das Irrationale erst der rationalen Bestimmung bedürftig ist. Diese leistet der Differtntialbegriff als Grundbegriff der intensiven Grössen. $\mathrm{Er}$ ist die Logisierung des Inhaltes, während die extensiven Kategorien, wie die der Zahl, die Logisierung der Form siud. Don hier aus gelangt Cohen zum Denkgesetz des Ursprunges, das Kinkel zum Gegenstand einer speziellen Untersuchung macht. Er untersucht die Beziehung dieses Gesetzes 
zum unendlichen Urteil und wandelt dieses Motiv durch geometrische Beispiele ab.

All diesen Aufsätzen ist, wie der Marburger Schule und ihren Begründern, die einseitige Verlegung des Schwerpunktes aus dem vorlogischen Material in die Sphäre logischer Bestimmung eigen. Es ist daher in keiner Weise einzusehen, inwiefern die letzten Triebfedern des Realismus durch diese Richtung paralysiert sein sollen.

Von den übrigen Aufsätzen der Kantstudien seien angefübrt: Bauchs Antrittsvorlesung "Immanuel Kant und sein Verhältnis zur Naturwissenschaft", Hönigswald „Zur Wissenschaftstheorie und -systematik“, Schultz „Über die Bedeutung von Vaihingers Philosophie des Als-Ob fïr die Erkenntnistheorie der Gegenwart", Münch „Das Problem der Geschichtsphilosophie“.

Bauch behandelt in einem vorzüglich disponierten Vortrag die mannigfachen Einflüsse, die von Kant auf die Naturforscbung ausgeübt wurden. Zunächst werden die Einzelleistungen gewürdigt, sodann die grundlegende Bedeutung, die er als Erkenntnistheoretiker für die Begriffsbildung des ganzen Gebietes gewann. Insbesondere die Grundlegung der Mathematik und ibre Beziehung zur Erfahrung; dann aber auch Kants Auffassung des Zweckbegriffes innerhalb der Biologie werden einer eingehenden Betrachtung unterzogen. Hier ist zumal die Parallele zwischen Kant und Darwin interessant. Beide erblickten ein fundamentales Problem in der seltsamen Übereinstimmıng, die zwischen der Gliederung der Begriffe und der Gliederung der Organismen in Arten und Gattungen besteht: Es ist das der Begreiflichkeit der Natur. Zwischen dem kontinuierlichen Aufbau der letzteren und der immanenten Gesetzmässigkeit des Denkens scheint eine tiefe Affinität zu bestehen, deren Erklärung letzten Endes ins Metaphysische auszulaufen scheint. Wenigstens deutet darauf auch Bruno Bauchs Hinweis auf die Kantische Konstruktion eines intellectus archetypus hin, der den Inhalt der Welt aus den Formen seines Denkens sich entfalten lässt, ihm daher die absolute Gesetzmässigkeit des letzteren einprägt.

Methodologischen Inhalts ist Hönigswalds Studie „Zur Wissenschaftstheorie und -systematik", in welcher der Verfasser zwischen den Auffassungen Rickerts und Cassirers in Bezug auf das Verhältnis der Logik zum Individuellen und zum Allgemeinen zu entscheiden versucht; beide Gesichtspunkte sollen einem noch un- 
fassenderen, einem Begriff des Objektes unterworfen werden, der das Prinzip des Wertes und der Wirklichkeit, mithin der naturwissenschaftlichen und der historischen Forschung in sich vereinigt.

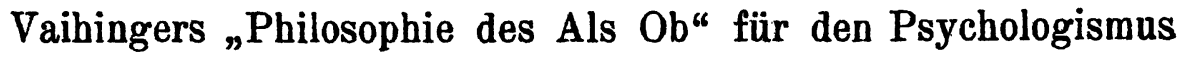
auszulegen, ist die Tendenz, die Schultz in seinem bereits angeführten Aufsatze verfolgt. Das Bindeglied bietet ihm der biolo= gistische Charakter der Fiktionenlehre, den ich freilich im vergangenen Jahresberichte in einem anderen, weniger dogmatischen Sinn zu deuten suchte. Der vorliegende Aufsatz beweist übrigens wie auch die mannigfachen Diskussionen, die sich bisher um den Pragmatismus bewegt haben, dass die Kontroverse zwischen dem logischen Absolutismus und dem psychologistischen Relativismus mit Aussicht auf Erfolg und eindeutige Endergebnisse sich lediglich an einer Frage orientieren kann, deren Beantwortung auch dem Neukantianismus Schwierigkeiten machte, der Frage nach der Anwendbarkeit der logischen Kategorien auf die Erfahrung.

Ähnlich wie Hönigswald betrachtet anch Münch in seiner gediegenen Studie „Das Problem der Geschichtsphilosophie“, letzteres als einen Spezialfall des Verhältnisses der Philosophie zur wissenschaftlichen Forschung. Der tiefste Grund geschichtsphilosophischer Begriffsbildungen muss in einem absoluten Werte gesucht werden, der sich seinerseits nicht deduzieren, sondern bloss unmittelbar erleben lässt. Nicht un ein individuelles Erlebnis handelt es sich hier aber, sondern um ein Weltsinnerlebnis, in welchem, als dem religiösen Grunderlebnis - wie vom Ver. fasser sehr richtig behauptet wird - alle Transzendentalphilosophie zuletzt veraukert bleibt.

Unter den Ergänzungsheften der Kantstudien hebe ich insbesondere Lanz "Das Problem der Gegenständlichkeit in der modernen .Logik“ hervor. Wir finden hier eine sorgfältige Darstellung dieses für die neue Erkenntnisphilosophie so zentralen Problems. Der Verfasser geht von Kants Theorie der Objektivität aus und stellt sodann unter den neueren Richtungen die psychologistische, die transzendente und die immanente Schule ein. ander gegenüber. Die Studie bietet eine tüchtige Bearbeitung. des vielverzweigten Materials. Hervorzuheben ist hier, dass ähnlich wie bei Külpe in der Charakteristik der immanenten Theorie der Gegenständlichkeit die Unterscheidungslinien zwischen dem Bewusstseinsidealismus und dem logischen Idealismus, welch letz. 
terer den Schwerpunkt vom Bewusstsein in den sachlichen Gehalt der Erkenntnis verlegt, deutlich gezogen werden.

Auch der vorkantische Philosoph Johann Nicolaus Tetens, von dem der Begründer des Transzendentalismus bekanntlich die Gliederung der Seelenvermögen und damit die Disposition zu seinen drei Kritiken übernommen hatte, bildet den Gegenstand eines von Dr. Wilhelm Uebele verfassten Ergänzungsheftes. Tetens erscheint hier weniger als ein Vorgänger des kritischen Kant, denn als Nachfolger des vorkritischen. Entscheidend hierfür ist sein Festhalten an der überkommenen Metaphysik, die Kant sowohl durch seine theoretische Kritik, als anch durch seine Verlegung des Schwerpunktes in die Ethik verdrängt hatte.

Die Kantgesellschaft hat auch die Neudrucke seltener philosophischer Werke fortgesetzt und um zwei interessante Bände bereichert. Otto Liebmanns bedeutungsvolle Schrift „Kant und die Epigonen“, die lange im Buchhandel gefehlt hat, ist, von Bruno Bauch herausgegeben, als zweiter Band erschienen. Programmatisch ist hier das Wiederanknüpfen an Kant unter strenger Ausscheidung aller Metaphysik des Ding an sich. Der Widerstand gegen das letztere ist freilich schon älteren Datums; und zwar muss man nicht etwa erst auf Fichte zurückgehen, von dem Liebmann zeigt, dass seine Weltanschauung in unverkennbarer Weise jenes Transzendieren der Erfahrung bekundet, das durch den Begriff des Ding an sich im allgemeinen bezeichuet ist. Entschiedener hat schon Salomon Maimon den Standpunkt der Bewusstseinsimmanenz vertreteu. Darüber unterrichtet der Neudruck Band III "Versuch einer neuen Logik" (XXVIII a. 445 S.), ein Werk, das in mancher Hinsicht die Positionen der Marburger Schule vorbereitet. So ist der Standpunkt der Bewusstseinsimmanenz weniger in bewusstseinsidealistischen, als im logistischen Sinne genommen. Schon im obersten Satze der Maimonschen Philosophie, im Grundsatz der Bestimmbarkeit gelangt dies zum Ausdruck. Durch diesen Satz wird nämlich gefordert, dass das Verhältnis des Ich zum Gegenstande ein allseitig bestimmtes sei, nach dem Schema von Subjekt und Prädikat im Urteile; womit die Annahme eines unbestimmbaren Dinges an sich hinfällig geworden ist. Die sorgfältige Analyse dieser Lehre, die Friedrich Kuntze in seinem Werk "Die Philosophie Salomon Maimons" (Heidelberg, Winter, XXV u. ,531 S.) vorgenommen hat, beweist übrigens, dass Maimon in seiner Auffassung der Transzendenz 
auch als Vorläufer Fichtes in Aubetracht kommt. Kuntzes Werk gibt eine erschöpfende Behandlung seines Gegenstandes, den es nach Erkenntnistheorie und Logik, Ethik und đ̈sthetik gliedert.

Nicht bloss der schon erwähnte Aufsatz Hönigswalds, sondern auch zwei andere Schriften dieses Autors beschäftigen sich mit Problemen der Methodologie: die Schrift „Zum Streit über die Grundlagen der Mathematik" (Verlag Winter, Heidelberg, 106 S.) und „Substanzbegriff und Funktionsbegriff, kritische Betrachtungen zu Ernst Cassirers gleichnamigem Werk" (Sonderabdruck aus der Deutschen Literaturzeitung Nr. 45, 46, 74 S.). Die Hauptfrage der erstgenannten Schrift ist die: Wie kann Mathematik auf die empirische Naturforschung Anwendung finden, ohne selbst auf empirische Grundlagen gestellt zu werden? Aber nicht allein die empirische Auffassung, sondern auch eine andere, die in modernen Theorien der Mathematik vielfach zum Ausdrucke gelangt ist, muss abgewehrt werden: die vom Willkïrcharakter mathematischer Konstruktionēn und Definitionen. „Nicht Willkür, diese besondere Form der subjektiven, psychologischen Evidenz, sondern eine im einzelnen Fall freilich unanalysierte Einsicht in ein System nathematischer, wissenschaftlich-objektiv gültiger Beziehungen, das heisst in die Natur des mathematischen Objektes ist es, was es erlaubt, zwischen einer, sei es begrenzten, sei es unbegrenzten Anzahl ron Möglichkeiten zum Zweck der Fixierung eines Ausgangspunktes mathematischer Deduktionen eine "beliebige" Auswahl zu treffen". Diese Auswahl ist frei, sofern sie nicht an die Bedingıngen eines äusseren Objekts gebunden ist. Ihre Freiheit ist indessen eingeschränkt durch die Eigenart des mathematischen Objekts selber, das heisst: durch den Hinblick auf den ganz bestimmten mathematischen Erkenutniszweck, dem eine Konstruktion zu dienen hat. Und so erscheint auch diese angebliche Willkür aus dem Bereich des Subjektiv-Psychologischen in die Sphäre gegenständlicher Bestimmtheit erhoben. Der Empirismus wieder beruft sich auf die Harmonie, die zwischen dem Naturlanfe und den mathematischen Werten besteht, eine Harmonie, durch welche die mathematische Naturwissenschaft ermöglicht wird. Indessen Natur und Mathematik - so wendet Hönigswald ein - bilden hier keine zwei getrennten Sphären, sondern die Naturauffassung selber ist schon durch Begriffe bedingt, die dem mathematischen Gebiete entstammen. Damit ist freilich das Problem bloss zurückgeschoben, denn es fragt sich jetzt, wie es 
denn überhaupt zu verstehen sei, dass der sinnliche Erfahrungsstoff unter ganz bestimmte übersinnliche Kategorien gestellt werden müsse. Von dieser Frage sahen wir ja, dass sie geeignet ist, den Rahmen des absoluten Logismus zu sprengen. Dies betont Hönigswald selber in seiner Kritik Cassirers, dessen Tendenz, alle gegenständliche Erkenntnis in das Schema mathematischer Funktionalbeziehung und Reihenbildung zu spannen, er in ihrer Einseitigkeit richtig beurteilt. Die verschiedenen Gegenstände erfordern besondere Behandlungsweisen. Unmöglich ist es, so heterogene Elemente wie mathematische, physikalische und psychologische an dem Faden einer einzigen Methode anfreihen zu wollen. In dieser Anerkenuung des Gegebenen, der Materie des Erkennens, greift Hönigswald auf den älteren Kritizismus zurück. Es scheint mir aber, dass damit nicht bloss der Rahmen des Logismus, sondern auch der des Phänomenalismus gesprengt ist. Die neuereu realistischen Versuche konnten sich auch mit Recht vor allem auf diese Abhängigkeit des kategorialen Systens vom irrationalen Inhalte der Welt berufen, der damit als ein Selbständiges legitimiert scheint. Seine ursprüngliche Denkfremdheit ist ja keineswegs aufgehoben, weil er, wie nicht zu leugnen, den kategiorialen Gesetzmässigkeiten des Denkens unterworfen wird.

Unter dem Einflusse der älteren Kantinterpretation Cohens ist Stadlers "Kants' 'l'eleologie“ entstanden, eine Schrift, von der eine unveränderte Neuausgabe vorliegt (Berlin, Dümmlers Verlagsbuchhandlung). Nicht allein die Erkenntnistheorie, sondern auch die Naturphilosophie ist an den hier erörterten Problemen beteiligt. Der Zweckbegriff ist ja das Bindeglieu beider Gebiete. Stadlers Untersuchung, die die transzendentale Betrachtungsart in möglichster Reinheit herausschälen will, zeigt die eigenartige Stellung, welche Kant zwischen Mechanismus und Vitalismus einnimmt, Wenn Kant die mechanistische Betrachtung als unzulänglich für die Erklärung des Organischen bezeichnet, so heisst dies nicht, dass er sie gelegentlich durch die teleologische ersetzen will. Eine solche Konkurrenz der Methoden besteht in Wahrbeit nicht; sie würde die systematische Einheit des Naturerkennens bedrohen. Der Mechanismus der Ursachen stellt ein sich geschlossenes System dar, das als Ganzes der Idee des Zwecks untergeordnet wird. So kann die letzter als Regulativ behilflich sein, unsere Kenntnis der Ursachen zu vervollkommnen, keineswegs aber, um ein anderes Erklärungsprinzip an ihre Stelle treten zu 
lassen. Ganz im Sinne der Ideenlehre wird demnach der metaphysische Zweckbegriff logisch umgedentet. Er ist nicht mehr die wirkende Macht in den Dingen, sondern bringt allein die systematische Einheit des Erkennens zum Ausdruck, so dass hier die Kluft, die für den reinen Transzendentalismus zwischen dem Sein und dem Denken besteht, vielleicht zu deutlichster Sichtbarkeit gelangt. Die moderne Auffassung strebt über die Relativität dieses Standpunktes hinaus. Soll sich die Zweckbetrachtung legitimieren, dann wird für sie dieselbe Bedeutung gefordert, die den Kategorien znkommt; dass sie nicht bloss ein Verfahren des Denkens, sondern eine eigene, objektiv begründete Seinsweise darstelle. Will mau diesen Kontrast $z$ wischen mechanistischer und teleologischer Betrachtungsart sich noch deutlicher vor Augen führen, so empfiehlt sich einerseits auch das Studium von Wundts "Prinzipien der mechanischen Naturlehre" (zweite umgearbeitete Auflage der Schrift: Die physikalischen Axiome und ihre Bėziehung zum Kausalprinzip, Stuttgart, Enke, XII u. 217 S.), andrerseits der Werke Driesch', dereń ich später Erwähnung geschehen lassen werde. Das Wesen des reinen Logismus, das Natorp in so knapper und treffender Weise dargestellt hat, wird nicht allein in der Behandlung logischer und erkenntnistheoretischer Fragen offenkundig, sondern auch anf den übrigen Gebieten der philosophischen Weltbetrachtung. Die. Vorzüge und Mängel dieser Methode enthüllen sich nicht minder in Ethik und Ästhetik. Beweis dafür ist Cohens „System der Philosophie“, dessen dritter Teil „Ästhetik des reinen Gefühls" (Berlin, Casirer, 1. Band XXV u. 401 S., 2. Band XV u. 477 S.) erschienen ist. Die erste Forderung, die der Verfasser erhebt, ist die der geschlossenen Systematik. Das System der Philosophie in einem seiner drei Glieder, Logik, Ethik, Ästhetik antasten, heisst es als Ganzes illusorisch machen. Der Begriff der Reinheit, den Cohen diesen organischen Erzeugungsweisen des Erkennens, Wollens und Fühlens zugrundelegt, ist in Sinne des strengen Transzendentalismus genommen und besagt, wenn man ihn in seine Elemente auflöst, wohl wesentlich dies, dass das philosophische Denken in Logik, Ethik und Ästhetik seine Begriffe selbständig erzeugen und entwickeln muss, ohne auf bestimmte Seinsinhalte - empirische oder metaphysische - zurückzugehen. Das ist ja, wie der programmatische Aufsatz Natorps gezeigt hat, die eigentliche Bedeutung dieser Richtung: das reine Denken darf den Rechtsgrund seiner Setzungen nicht in einer Realität ausser- 
halb seiner suchen, da es eine solche gar nicht gibt; da vielmehr alles, was den Anspruch auf Realität erhebt, erst von den Mitteln des Denkens aufgebaut wird. Es sind hier aber zwei Motive auseinanderzuhalten, die in der Darstellung Cohens nicht getrennt werden: das Motiv der Systembildung und das Motiv des Logismus. Jede Metaphysik der Kunst wird als eine romantische Reaktion gegen die systematische Philosophie verworfen. Mag man die Notwendigkeit des Systems aus der Einheit der Kultur auch zugestehen, mag man in der nachdruicklichen Betonung und Darstellung dieses Zusammenhanges, wie billig, ein besonderes Verdienst Cohens und seiner.Schule rühmen; mag man demgemäss alle Absplitterung und Aphoristik des Gedankens, wie sie eine Gefahr des modernen Geistes bildet, ablehnen, der Begriff des Systems scheint mir hier willkürlich gefasst. Es ist nicht einzusehen, warum durch ihn eine metaphysische Weltbetrachtung von Anbeginn ausgeschlossen sein soll. Freilich, wenn durch das künstlerische Schauen und Gestalten das ethische Verhalten ersetzt und verdrängt wird, wie dies bei manchen Romantikern geschieht, dann mag dies Willkür uud Phantastik genannt werden. Weshalb aber sollte Metaphysik mit einem systematischen Betriebe nicht vereinbar sein? Ja, weshalb sollte der letztere eine unbedingte Koordination seiner Glieder, Ethik, Logik und Ästhetik, fordern und nicht auch einer Über- und Unterordnung derselben Raum geben, vorausgesetzt, dass sie nicht eigenmächtig behauptet werde, sondern aus einem einheitlichen Grundgedanken sich ergebe? Unverkennbar wird der dogmatische Ausgangspunkt, wenn man erwägt, wie in diesem System die Religion zu kurz kommt. Was ist das Kriterium dafür, dass sie nicht als selbständiges Glied neben den anderen behandelt wird? Wie es scheint die Dreiteilung des geistigen Universtims in die Sphären des Erkennens, Fühlens und Wollens. Aber davon abgesehen, dass diese Einteilung der psychologischen Realität entnommen ist, lässt sich ihre unbedingte Stringenz nicht begreiflich machen. Die Möglichkeit eines anderen Einteilungsgrundes, welcher der religiösen Funktion einen sèlbständigen Ort eingeräumt hätte, ist nicht von der Hand zu weisen. Hier kommt dem systematischen Bedürfnis das logistische in die Quere. Weil das religiöse Empfinden noch deutlicher als das ethische und ästhetische seine Beziehung zur Metaphysik enthüllt, soll es zu einer Unterart anderer Funktionen nivelliert, werden. 
Natïrlich wird damit das ausserordentliche Verdienst dieser Arbeit ebensowenig wie das der früheren bestritten. Nicht allein die Feinheit der begrifflichen Konstruktion, die erstaunliche Elastizität des Gedankens muss gerühmt werden, sondern auch die subtile Analyse mancher künstlerischen Erscheinungen. Für die Grundlegung der Ästhetik ist die Bestimmung des Verhältnisses der drei Richtungen des Bewusstseins zum Ichproblem massgebend. Das erkennende Bewusstsein geht darin auf, Erzeugung des Objekts zu sein; die Einheit des Selbstbewusstseins ist für dasselbe nicht von Bedeutung. Aber auch im wollenden, ethischen Bewusstsein ist das Ich als solches nicht gegeben, sondern lediglich als Allheit, als universales Selbst, das in der Idee der Humanität seine Verklärung findet. Erst das ästhetische Bewusstsein steuert auf ein Selbst hin, welches das Individuum schlechthin zur Aufgabe macht. Diese Geburt der reinen Individualität vollzieht sich im Liebesgefühle, welches das Beherrschende des künstlerischen Schaffens und Schauens ist.

Andere Wege geht Broder Christiansen in seiner „Philosophie der Kunst“" (Verlag von Klaus und Feddersen, Hanau). Obgleich auch dieser Denker dem engeren Kreise des Transzendentalismus angehört, schliesst sich seine Problemstellung - in mancher Hinsicht nicht unähnlich der des Ästbetikers Guyau und der neueren französischen Schule - enger an die Wirklichkeit an, und zwar an die Wirklicbkeit des Lebens. Kunst ist Selbstoffenbarung des Absoluten und Metaphysischen im Menschen. Auch in Broder Christiansens „Kritik der Kantischen Erkenntnislehre" (Verlag von Behr, Berlin, 177 S.) vorspürt man die Tendenz, über die Enge des reinen Logismus hinauszukommen. Die Schrift scheint mir freilich in ihren kritischen Auseinandersetzungen wertvoller, als in ibrem positiven Ertrage. Der Verfasser zeigt, in diesem entscheidenden Punkte mit Leonard Nelson übereiustimmend, dass das Problem der Erfahrung von Kant und den Kantianern nicht iu richtigen Sinne gestellt ist. Man darf es nicht aus dem wissenschaftlichen Denken deduzieren, denn dieses muss ja erst kritische Rechtfertigung finden. Und ebensowenig darf man es auf die transzendental-psychologische Organisation des Subjektes zurückfiihren, da ja sonst gegen den wahren Geist des Transzendentalismus die quaestio juris zur quaestio facti herabgewertet wird. Das Problem der Erfahrung muss als ein wertkritisches formuliert werden. Aus der Definition der Realität sind 
die Kategorien abzuleiten; wie diese Ableitung sich im näheren zu vollziehen habe, lässt der Verfasser freilich unbestinmt. Auch Christiansen glaubt wie andere moderne Erkenntnistlleoretiker den Richtpunkt für die Bildung des Realitätsbegriffes in einem Sollen zu finden, welches er freilich konkreter bestimmt als Rickert und seine Schüler. Völlig klar hat er sich darüber noch nicht ausgesprochen; und es ist vielleicht von einer Fortsetzung des Werkes die deutliche Ausgestaltung dieses Gedankens zu erwarten. Der Realitätswert wird auf dem Wege des Sollens in Beziehung zu einem metaphysischen Trieb des Subjekts gebracht. Welcher Art aber dieser Trieb ist, wie er sich entfalte und wie mit inm die strenge Objektivität des Wertes vereinbar sei, findet sich kaum angedeutet. Und dennoch könnte dies ein Punkt sein, vou dem aus eine entscheidende Wendung im Verhältnis von Erkenntnistheorie und Metaphysik sich zn ergeben vermöchte.

Obgleich Christiansen den reinen Transzendentalismus vertritt, lässt er die Möglichkeit einer metaphysischen Realität und metaphysischer Erkenntnisse zu. Aber auch diese Erkenntnis müsste eine empirische sein; sie liesse sich weder in analytischen noch in synthetischen Urteilen a priori darstellen.

Als eine Ergänzung kann Christiansens Schrift „Vom Selbstbewusstsein" (Verlag Friedrich Feddersen, Berlin, 87 S.) angesehen werden. Auf Grund scharfsinniger Analysen will der Verfasser hier zu den überraschenden Resultat gelangen, dass es ein unnittelbares Wissen vom Seelischen, eine Unmittelbarkeit des Selbstbewusstseins nicht gibt, dass nicht Anschauung, vielmehr Reflexion das Werkzeug der Selbsterfahrung ist. Die Tiefen des Seelenlebens bleiben nuerkannt. Einen Beweis dafür sucht der Verfasser in der Tatsache, dass die Psychologie ungeachtet einer Bemühung von mehr als zweitausend Jahren nicht imstande ist, die elementaren Vorgänge der Innenwelt in widerspruchsfreier Weise aufzuzeichnen.

$\mathrm{Zu}$ einem wesentlich anderen Ergebnisse für die psychologische Erkenntnis gelangt Walther Schmied-Kowarzik in seiner Schrift "Umriss einer neuen analytischen Psychologie“ (Leipzig, Ambrosius Barth, VI n. 318 S.). Er will der Psychologie apodiktische Erkenntnisse entringen, die prinzipiell auf der gleichen Stufe stehen wie die logischen oder mathematischen. Die Problemstellung ist hier zweifellos eine originelle. Der Begriff der Analyse ist in einem neuen, wesentlich von Kant abweichenden Sinne 
gefasst. Er wird nicht mehr dem der Synthese kontrastierend gegenübergestellt, sondern dem der Feststellung empirischer Tatsächlichkeiten. Der Verfasser ist darin im Rechte, dass er den prinzipiellen Gegensatz von Analyse und Synthese aufhebt, da es sich hier eher um verschiedene Richtungen eines und desselben Sachverhaltes handelt. Die Aualyse, so könnte man sagen, ist die rückwärts geschaute Synthese; sie ist die Auflösung irgendeines gegebenen Komplexes in seine Elemente, die Einsicht in deren inuere Beziehungen. Da für diese Einsicht die Existenz des Komplexes gleichgiltig ist, eignet ihr das Merkmal der Daseinsfreibeit. So gewinnen wir durch Aualyse des mathematischen Raumes die geometrischen Gesetze, deren absoluter Wert nicht von der Existenz der ihr zugruudegelegten Gebilde abhängig ist; in ähnlicher Weise können wir nach Ansicht des Verfassers durch das Prinzip der Analyse in der Psychologie zu apodiktischen Erkenntnissey gelangen und so. Diltheys Forderung einer beschreibenden und zergliedernden Psychologie durch eine strenge Systematik des Seeleulebens erfüllen.

So einlenchtend diese Ausführungen in mancher Hinsicht sind, es scheint mir, dass die Eigenart der analysierten Komplexe in ihnen zu wenig berücksichtigt wird. Denn zweifellos besteht ein prinzipieller Unterschied zwischen einem Komplex, der wie der mathematisch-mechanische selber ans idealen, daseinsfreien. Elementen gebildet ist, und einem Komplex, der wie der psychologische aus empirischen Elementen besteht. Die Analyse des letzteren ergibt höchstens Gleichheit und Verschiedenheit, ohne das Ausmass dieser Relationen in absoluter Weise bestimmen zn können. Daraus erklärt sich ja die mechanistische Reduktion der Qualitäten auf Quantitäten durch die Naturforschung. Dazu kommt weiter, dass das Seelenleben, wie vom Verfasser selber betont wird, ein unaufhörlicher, einheitlicher Prozess ist, dessen Elemente fluktuierend sind und daher schwerer unterschieden werden können als die der Aussenwelt.

Jit der gleichen Schwierigkeit ist Rehmkes logistische Darstellung der Seelenvorgänge in der Schrift "Die Willensfreiheit" behaftet (Verlag Quelle \& Neyer, Leipzig, 146 S.). Rehmkes Begriffsbestimmungen sind bier wie überall ausserordentlich präzis. Eben deshalb scheinen sie mir aber nicht völlig geeignet, das fluktuierende Element des Seelischen in sich zur Darstellung zu bringeu. Das Verfahren ist hier ein allzu definitorisches; während 
es das Wesen der seelischen Wirklichkeit ist, oft zwischen den Gliedern einer schroff gespannten Alternative in der Mitte hindurcbzugleiten. Die Lösungsversuche des Determinismus und des Indeterminismus werden auch von Rehmke als in ihren Voraussetzungen unhaltbar beiseite geschoben. Seine eigene Lösung hängt an der aus seinen früheren Werken bekannten Anuahme, dass der Wille die sich ursächlich selbst beziehende Seele, und dass die Seele ein besonderes Einzelwesen ist. Es wird zwischen Wahlwollen und Zwangsvollen unterschieden, die aber beide unter dem Zeichen der Selbstbestimmung stehen. „Inmer aber ist es das Bewusstsein als Wille, immer also ein Wille, was sich selbst bestimnt; und immer ist das, zu dem ein Wille sich selbst bestimmt, ein besonderes Wollen, und zwar sei es das Wollen einer Erweiterung oder einer Besonderung des ursprünglich Gewollten - so beim freien, oder, was dasselbe trifft, beim Wahlwollen sei es bloss das Wollen einer Erweiterung des ursprünglichen Zweckes - so beim Zwangwollen". Willensfreileit und Willenszwang stehn in einem Gegensatz, nicht aber Willensfreiheit und Willensnotwendigkeit.

Die analytisch-psychologische Betrachtungsweise ist, wie Schmied-Kowarzik richtig hervorhebt, verwandt der phänomenologischen, deren sich Husserls "Logische Studien", zumal im zweiten Bande, bedienen. Auch Wilhelm Schapps "Beiträge zur Phänomenologie der Wahrnehmung" (Max Niemeyer, Halle a. S., 157 S.) bewegen sich in der von Husserl bezeichneten Richtung. Im Gegensatze zum impressionistischen Empirismus will die $\mathrm{Phäno-}$ meuologie die Art des Gegebenseins jener Werte feststellen, die sich nicht in pure Inıpressionen auflösen lassen. Dahin gehören zum Beispiel die ontologischen und logischen Grundwerte, die Kategorien der Identität, der Substanz, die mathematischen Begriffe. Aber auch die Wahrnehmung bedarf dieser phänomenologischen Analyse, denn in ihr sind, dem landlänfigen Empirismus entgegen, dessen Voraussetzungen sehr häufig auch von den rationalistischen Erkenntnistheorien aufrecht erhalten werden, heterogene Bestandteile vereint. Nicht allein die Unterscheidung des Inhaltes der Empfindung von ihrer Form, sondern auch die Mitwirkung der Idee ist hier zu beobachten. An ihr ist es gelegen, dass wir in der Wahrnehmung nicht bloss etwas anschauen, sondern auch etwas meinen. Im Grunde länft dies darauf hinaus, dass neben der individuellen Besonderheit des allgemein Gegebenen 
sich darin auch ein Gattungsmässiges, Allgemeines ausspricht. Es ist dies die elementarste Form jener Anteilnahme an der Idee, die schon Plato den Erscheinungen zuerkannt hatte. Es bedarf kaum einer Erwähnung, dass dieser Zusammenhang auch für das Kategorienproblem von Bedentung ist. Die schroffe äussere Trennung von Erscheinungen und Kategorien, die auf jene angewendet werden, eine Trennung, gegen die der Neukantianismus sich nit Recht wendet, wird auch von dieser Seite aufgehoben. Man muss, da die Erscheinung selber kategorial bestimmt ist, den Gegeusatz zwischen Rationalem und Irrationalem, unmittelbar Gelebtem und Reflektiertem in sie hineinverlegen. Übrigens ist es ein Verdienst Rehmkes, in seiner Philosophie auf die Gegebenheit des Kategorialen nachdrücklich hingewiesen zu haben.

Einer enzyklopädischen Darstellung der Logik begegnen wir im ersten Bande der von Ruge im Verein mit Windelband herausgegebenen Enzyklopädie der philosophischen Wissenschaft (Tïbingen, Verlag von Mohr, 1912). Deu Zweck des Unternehmens formuliert der Herausgeber in der Einleitung: es ist der einer systematischen Einheit der Erkenntnisse unter möglichster Vermeidung persönlicher Einseitigkeit. Aus letzterem Grunde kommen verschiedene Denker als Repräsentanten verschiedener Nationen und Richtungen zu Worte: Windelband, Royce, Couturat, Croce, Enriques, Lusskij. Windelband. teilt das Gebiet in formale Logik und Methodologie ein. Die obersten Denkgesetze der formalen Logik sind nicht lediglich Normen für ein erkennendes Bewusstsein; dies ergibt erst ihre Anwendung auf die psychologische Sphäre, von der man ihre autonome, in sich berubende Bedeutung zu unterscheiden hat. Es sind auseinanderzuhalten die Geltung an sich und die Geltung für uns. Diese Anerkennung der reinen Gegenständlichkeit des Logischen, diese Trennung des Wertes von der Norm ist ein bedeutungsvolles Motiv der modernen Erkenntnislehre, das uns auch bei Rickert, Lask und Meinong begegnet. In der Erläuterung der obersten Denkgesetze will Windelband diesem Prinzip Rechnung tragen. So besagt der Satz vom IViderspruche, dass Bejahung und Verneinung derselben Beziehung nicht zugleich gelten können. Er fordert den vicht seltenen psychologischen Schwankungen gegenüber eine eindeutige Entscheidung. Noch klarer markiert der Satz vom ausgeschlossenen Dritten die Grenze zwischen dem logischen Wert und seiner psychologischen Anwendung, sofern aus ihm überhaupt keine Norm sich ableiten 
lässt; wenn er nämlich feststellt, dass Bejahung und Verneinuug desselben nicht beide falsch sein können, so ist das empirische Bewusstsein gleichwohl nicht immer in der Lage, sich für das cine oder das andere zu entscheiden. Hier tritt ein neues Denkgesetz in Funktion, das des zureichenden Grundes, welches gebietet, dass jede Behauptung, positive oder negative, nicht willkürlich, sondern sachlich begründet auftrete. In der Kategorienlehre geht Windelband auf seine Unterscheidung in reflexive und konstitutive Kategorien zurück. Konstitutiv sind die Kategorien, die als wirkliche Verhältnisse zwischen den Gegenständen gedacht werden; reflexiv jene, die - wiewohl durch die Eigenart der Gegenstände bedingt - dennoch erst im Bewusstsein und für das Bewusstsein bestehen. Das reflektierende Bewusstsein ist vergleichend und unterscheidend und als solches Fundament der mathematischen diskursiven Kategorie. Die konstitutiven Kategorien bilden die transzendentale Logik; sie treten durch Raum und Zeit in unmittelbaren Zusammenhang mit der objektiven Realität. In der Methodologie wird das Verhältnis von Induktion und Deduktion, von idiographischer, historischer und nomothetischer, naturgesetzlicher Methode in den Mittelpunkt gestellt. Das eigentliche Element der Methodologie ist die Hypothese, die der Tatsachenvorstellung zugrundegelegt, rückwirkend in ihr erst ihre Bewährung findet. Den letzten T'eil der Abhandlung bezeichnet die Erkenntuistheorie, in der das Verhältnis les Seins zum Gelten als einer nicht weiter auflösbaren Relation: Form und Inhalt bestimmt wird. Von der Logik zur Metaphysik führt kein geebneter Weg. Das Erkennen ist eine Auslese aus einer reicheren Totalität, die sich demnach zu seinen Inhalten verbält nicht wie das Ding zur Erscheinung, sondern wie das Ganze zum Teil.

Bei Royce hat die Logik dèn Charakter einer allgemeinen Ordnungslehre, in deren Ausgestaltung er eine kritische Synthese der rationalistischen und voluntaristischen Betrachtung erstrebt. Die Wahrheit ist rational und insoweit absolat, als sie das Prinzip der Selbstgarantie in Anspruch nehmen darf. Sie darf dies überall dort, wo der Versuch, von ibr zu abstrahieren, eben ihre Unaufhebbarkeit zutagetreten lässt. Nicht allein in Satz der Ideutität verhält es sich so, sondern auch in Leitprinzip der Ordnungslehre, dem Einteilungsprinzip der Klassen und Relationen. IVer das Vorhandensein von logischen Klassen bestreitet, lässt ausser Acht, dass er damit selber eine Klassifikation vorgenounmen hat. 
Desgleichen setzt, wer das Vorhandensein von Relationen leugnet, in dem Urteil, das solche Leugnung enthält, selbst eine Relation. Bleibt somit die logische Sphäre ein in sich beschlossenes, störenden Eingriffen von aussen enthobenes System, so kann man, ähnlich wie dies bei Fichte erscheint, dem Systemganzen dennoch ein Willensprinzip zugrundelegen. Einzig und allein auf diesem von Royce betretenen Wege ist ein Ausgleich zwischen den berechtigten Forderungen des Transzendentalismus und des Pragmatismus möglich.

Über die Verbindung von Logik und Mathematik, die del Algorithmus bezweckt, äussert sich Couturat, der die Unabhängigkeit der Logik von der Mathematik betont und bloss die Notwendigkeit eines allgemein rechnenden Verfahrens hervorhebt. In seiner Theorie der Definitionen und Demonstrationen vertritt er eine geistvolle Synthese absoluter und relativer Aspekte, die man als methodologischen Perspektivismus bezeichnen kann. Die Auswahl der obersten Begriffe, die der Definition, und der obersten Sätze, die der Demonstration zugrundegelegt werden, die Auswahl der Axiome und Theoreme hängt an der Willkür des Ausgangspunktes, ist sonach subjektiv. Die unter dieser Annahme sich ergebende Verknüpfung von Begriffen und Sätzen behält dessenungeachtet ihre objektive Bedeutung. Was Problem einer internationalen Sprachbildung bietet Couturat den Anlass, den Nominalismus, der die Priorität des sprachlichen Ausdruckes vor dem logischen Gehalte anninmt, wirksam zu widerlegen. Jenes Problem enthält ja die Weisung, die logischen Grundmotive, die der Einfachheit und Eindeutigkeit, welche durch die bestehenden Sprachen bloss unvollkommen erfüllt werden, zur bewussten Grundlage eines neueu, künstlichen Idions zu machen.

Ist Couturat Formalist, so fällt für den Hegelianer Benedetto Croce Form und Inhalt des Denkens, Erkenntuislehre und Metaphysik zusanmen. Als konkrete Idee ist das Denken zugleich Setzung von Realem. Die Trennung der reinen von der angewandteu Logik wird nach diesem Prinzipe als leere Abstraktion verworfen. Die äusserliche Zweiheit des Logischen und des Realen gibt es nicht und dementsprechend anch keine mechanische Anwendung des einen auf das andere, sondern lediglich eine organische Entwicklung des Logischen am Stoff der Realität. Die vier entwickelten Formen des Wissens: Dichtkunst, Philosophie“ Naturforschung und Nathematik sind begründet in den Formen 
der Vorstellung, der Idee, des klassifikatorischen und des abstrakten Begriffes. Das naturwisseuschaftliche und mathematische Erkennen fusst mithin auf technischeu Fiktionen, während die Philosophie den vollen Gehalt der. Wirklichkeit ergreift und so auch religiöse und geschichtliche Erkenntuis in sich anflöst.

In Gegensatz zu Benedetto Croce geht Federigo Enriques auf die Unterscheidung zwischen Logischem und Wirklichem zlrück und sieht sich somit abermals vor das Problem des Verhältnisses beider gestellt. Die logischen Grundsätze sind nicht der Veränderung unterworfen, die das Wesen der sinnlichen Wirklichkeit bildet. Bloss iusoferne kommt die letztere dem Rationalen näher, als-sich in ihr langsam wechselnde Elemente finden, die zu logischen Gegenständen geprägt werden können, als mithin der Prozess einer gradweisen Annäherung stattfindet. Die Realität ist demnach nicht selber logisch; sie weist indessen eine 'Tendenz auf, es zu sein. Es ist klar, dass der wechselnden Charakteristik dieses Verhältnisses nicht so sehr ein verschiedener Begriff des Renlen als dus Logischen zugrundegelegt ist; für Croce ist Gasselbe absolute Bewegung, für Enriques und die durch ihn repräsentierte Richtung festgefügte Form.

Die Umgestaltung des Bewusstseinsbegriffes in der modernen Erkenntnislehre und ihr Ergebnis für die Logik ist der Gegenstand der letzten von Nicolai Losskij verfassten Studie. Sein Standpunkt ist ans dem Werke "Grundlegung des Intuitivismus" bekannt. Das Bewusstsein ist nichts Psychologisches oder Subjektives; und so stehen Bewusstscinsinhalt und objektive Realität in keinem Verhältnis kontradiktorischer Gegensätzlichleeit. Ähnlich ist ja der Berwusstscinsbegriff schon von Denkern wie Schuppe, Lipps, Rehmke gedeutet worden, zwischen denen freilich wieder bedentende Unterschiede besteben, Subjektiv ist der Akt der Erkenutnis, nicht ihr Gegenstand. Die Analyse druickt die subjektive, die Synthese die objektive Seite der Erkenntnisfunktion aus. Denn alles Analysieren ist ein Vergleichen und das Vergleichen ein psychischer Vorgang. Das Verglichene aber stellt eine synthetische Einheit, einen objektiven logischen Zusanmenhang dar. Analytisch ist der Satz der Identität, synthetisch der Satz vom zureichenden Grunde. Das Identitätsprinzip besitzt eine bloss regulative $\mathrm{Be}$ deutung, der Satz vom zureichenden Grunde beherrscht das positive, im Urteilen und Schliessen sich vollziehende Erkennen. 
Ein Hauptproblem der Logik behandelt Hans Pichler „Möglichkeit und Widerspruchslosigkeit" (Leipzig, Verlag Ambrosius Barth, 72 S.). Der scharfsinnigen und exakten Darstellung Pichlers erweitert sich das liöglichkeitsproblenı dermassen, dass es auch das Problem der Apriorität und Aposteriorität in sich fasst. Wie in seinen früheren Schriften änssert der Verfasser auch hier die Tendenz, vom Kantischen zum Leibnizschen Gedankenkreise zurückzukehren. Letzterer liegt auch wirklich mebr in der Richtung jener rein objektivischen Erkenntnisweise, die hier zum Ideal erhoben wird. Ihr Wesen ist die daseinsfreie, von aller Anwendung aufs Empirische losgelöste Bestimmung der Kategorien als solcher. So wird auch hier der Nachweis unternommen, dass Leibniz' logischer Möglichkeitsbegriff, dessen Merkmal Widerspruchslosigkeit ist, durch Kants Kritik nicht aufgelöst wurde. Die Einschränkung des logischen liöglichkeitsbegriffes, sein Abstand von der objektiven Möglichkeit hängt aufs innigste zusammen mit der Relativität menschlichen Erkennens, so dass sich eben an diesem Punkte die Denkbarkeit einer höheren Form der Intellektualität eröffnet.

Die von Gerhard Hessenberg und Leonard Nelson herausgegebenen Abbandlungen veröffentlichen unter anderem den Vortrag, den Nelson auf dem Kongress zu Bologna über die Unmöglichkeit der Erkenntnistheorie gehalten hat. Dieser Vortrag fasst die Ergebnisse seines grösseren Werkes in übersichtlicher Weise zusammen. Die Erkeuntnis ist ein Faktum, das nicht zum Problem gemacht werden kann; denn es zum Problem machen heisst, ein Kriterium der Erkeuntnis suchen, welches bloss in einer anderen Erkenntnis gefunden werden könnte, womit die Fragestellnng ins Endlose zurückgeschoben wäre. Das Faktum der Erkenntnis vollzieht sich aber nicht in Urteilen, die als solche durch Reflexion vermittelt sind: es stellt sich vielmehr als ein Unmittelbares dar, das indessen nicht anschaulicher Art sein soll. Der Weg zur Feststellung dieser Erkenutuis ist die psychologische Zergliederung, die sie allerdings bloss aufzeigt, nicht aber begründet. Durch letztere Einschränkung entkräftet Nelson den Vorwurf des Psychologismus, der von logistischer Seite gegen inn erhoben wurde. Dass der Nachweis der Kategorien letzten Endes bloss ein psychologischer sein kann, wird man Nelson zugeben müssen. Weniger glücklich scheint mir seine Widerlegung der intuitionistischen Erkenntnislehre. Diese könnte erst gelingen, 
wenn wirklich gezeigt würde, dass die Schranke zwischen Anschaunng und Begriff eine unaufhebbare ist. I)ie Unvereinbarkeit beider ist indessen für die moderne Philosophie eher ein Dogma, als eine berviesene Behauptung.

Als ein interessantes Gegenstück zur Nelsonschen Theorie mag eiu anderer Aufsatz betrachtet werden, der Kastil zum Verfasser hat: "Jakob Friedrich Fries' Lehre von der unmittelbaren Erkenntnis." Von denselben Voraussetzungen gelangt er zum entgegengesetzten Resultat. Mit Nelson stimmt er darin überein, dass alle transzendental-logischen Versuche des Nachweises der Kategorien uuzulänglich sind. Während aber Nelson mit Kant annimmt, dass der Weg der inneren Erfahiung der richtige ist, will Kastil anch die Unzulänglichkeit des letzteren beweisen. Die innere Frfahrung zeige uns nämlich, dass metaphysische Urteile anch blind gefällt werden können, ohne dass sich eiu Kriterium ihres Unterschiedes von einsichtigen Erkenutnissen biete.

Nit dem Erkenntnisproblem aufs engste verknüpft ist die Theorie des Urteils, das innerhalb des Kantianismus zumeist als Grundforn des Erkennens charakterisiert wurde. In heftiger Opposition gegen diese Anffassung fiuden wir vor allem Nelson, der ein unnittelbares Erkenntuisprinzip fordert; während alles Urteilen auf Vermittlung und Reflexion beruhe. Von wesentlich anderen Ausgangspunkten gelangt Lask in seiuer "Lehre vom Urteil“ (Tübingen, Verlag Nohr, VII u. 207 S.) zu einem ähnlichen Ergebnisse. Das Urteil gehört nicht in die Sphäre des Gegenstäudlich-I Logischen, wodurch objektive Erkenntnis konstituiert wird; sondern in die eines nachbildenden, daher subjektiv gefärbten Verhaltens. Wegweiscnd für diese Urteilstheorie waren Rickerts Untersuchungen des Urteils, die namentlich die Unterscheidung seiner psychologischen und logischen Seite vollzogen. Zumal in dem Aufsatz des Logos (Band III, Heft 2) „Urteil und Urteilen" hat Rickert die Gliederung des Urteils in die psychische Urteilswirklichkeit, in den transzendenten und den immanenten Sinn, durchgeführt. Der transzendente Urteilssiun ist der objektive logische Gehalt, das darin gegenständlich Bejahte oder Verneinte. Es ist dasjenige, dessen Zusammengehörigkeit oder Getrenntheit eben im Urteile zur Aussage gelangt. Dieser transzendente Sinn kann aber dem Subjekte nicht völlig transzendent bleibeu, wenn es ihn überhaupt aussagen, heurteilen soll. Es muss auf ihn gerichtet sein, muss im Urteilsakt zu ihm Stellung 
nehmen. „Dem gültigen Wertgehalt oder dem Sollen im Objektiven muss ein wertendes Anerkennen oder Bejahen im Subjektiven entsprechen." Auch dieses Verhalten aber muss als ein aus dem rein zeitlichen Verlauf des psychischen Urteilsaktes heraustretendes, weil auf gïltige Werte eingestelltes betrachtet werden. Mit Rickerts Unterscheidungen hängt Lasks Analyse des Urteils zusammen. Lask steht hier, wie in seiner "Logik der Philosophie" völlig anf dem Boden des Transzendentalismus. Gegenständliches Erkennen muss vọ allem nachbildenden Erkennen getrennt werden. Urteilen aber beisst, ein scbon Gegebenes nachbilden. Das gegenständliche Sein gliedert sich in Kategorien und Kategorienmaterial; das heisst, die irrationalen Inbalte müssen, un gegenständliche Bedeutung zu erhalten, unter bestimmten Kategorien stehen. Diese gegenständliche Sphäre ist die Sphäre der Gegensatzlosigkeit. Dadurch unterscheidet sie sich am deutlichsten vom Urteil, das auf dem Gegensatz von Bejahen und Verneinen beruht. Im Urteile aber bedarf es - und hier ist die Verwandtschaft mit Rickert unverkennbar - zweier Gegensatzpaare. Dis Gegensätzlichkeit von Richtigkeit und Falschheit genügt nicht. Dass etwas richtig oder unrichtig ist, dafür muss es einen objektiven Grund in der Zusammengehörigkeit oder Nichtzusammengehörigkeit der verbundenen oder getrennten Elemente geben. Dieser Gegensatz wird von Lask als der von Wahrheit und Wahrheitswidrigkeit bezeichnet. Solch ein gegensätzliches Verbalten bezeugt eine Relativität und kann deshalb nicht in den absoluten Gegenstand der Erkenntnis verlegt werden. In diesem gibt es es blos ein schlichtes Ineinander von Kategorien und Kategorienmaterial. Erst indem die erkennende Subjektivität unsicher und schwankend an den gegenständlichen Sachverhalt herantritt, entsteht jene Spaltung und Zerstückung desselben, die sich in der Wertgegensätzlichkeit bekundet. An sich repräsentiert er einen übergegẹnständlichen Wert. Innerhalb des Logischen selber muss auf diese Veise zwischen dem im strengen Sinn Objektiven und dem Eingreifen der Subjektivität unterschieden werden.

Hit dem Zusanmenhang von Wert und Wirklichkeit befasst sich eine lesenswerte Schrift von Nicolai von Bubnoff "Zeitlichkeit und Zeitlosigkeit" (Heidelberg, Winter, 65 S.). Es wird hier gezeigt, wie der Gegensatz der Seinslehre und der Werdenslehre die antiken Problemstellungen überdauert hat und bis in die neuesten Theorien der Erkenntnis und des Wertes hineinreicht. 
Besonders wichtig ist die Feststellung, dass die Gegensatzpaare des modernen Logismus Sein und Gelten, reales und ideales Sein, Sinnliches und Unsinnliches, Wirklichkeit und Wert, sich nicht iiberall mit dem fundamentalen Gegensatz von Zeitlichkeit und Zeitlosigkeit decken: dass das Verhältnis vielmehr ein komplizierteres, mannigfachen Schwankungen unterworfenes ist. In ziemlicher Reinheit lebt der Heraklitismus wieder in der Intuitionsphilosophie Bergsons auf. Auf die erkenntnistheoretischen Schwierigkeiten der letzteren, die sich in dem logisch wicht fasslichen Begriff der Intuition verbergen, wird mit Recht hingewiesen. Sehr richtig ist ferner die Bemerkung, dass der Weg der Philosophie von der ursprünglichen Einheit des undifferenzierten Erlebens durch die Wertanalyse hindurch zur differenzierten Einheit einer systematischen Weltanschauung, von der Mystik durch die Wertanalyse zur Metaphysik geht. Die zukünftige Philosophie wird, sofern sie eine Synthese von Intuition und Logizität erstrebt, sich dieses Motivs zn bemächtigen haben.

Als Studien wider Hegel und die Kantianer bezeichnet Hans Ehrenberg seine schwierige, aber interessante Schrift „Die Parteiung der Philosophie" (Leipzig, Meiner, IV u. 133 S.). Ehrenberg unterscheidet dreierlei: die Logik, die Disziplinen und das Absolute. Der Fehler Hegels. und des Kantianisnus sei es gewesen, dass beide die Einheit der drei Sphären in die Logik gesetzt haben; der Kantianismus, indem er den Primat der Logik auf ihre Stellung zu den Disziplinen, Hegel, indem er ibren Primat anf ihre Stellung zum Absoluten gründete. Daher wurden die Disziplinen von Hegel vergewaltigt, das Absolute von Kant uegativ beiseitegesetzt. „In jedem Falle ist das, was uns unter dem Namen einer Logik entgegentritt, irgendwie die Antizipation des Systems; was man von Hegel gesagt hat: ,Die Logik blieb das esoterische System, und das System die exoterische Logik', gilt - mutatis mutandis - auch vom Kautianismus. Weun wir uns die Befreiung des Systems von der Torberrschaft der Logik zum Ziel gesetzt haben, so bekämpfen wir in ganz gleicher IVeise Hegelianismus und Kantianismus. Den Primat der Logik zu vernichten, wenden wir uns jetzt der Harmonisierung der Dreiheit Logik, Disziplinen, Absolutes zu.“ Eben die völlige Reinigung der Logik, ihre im Hegelschen Sinne vorgenommene Entmaterialisierung, bringt auch die Befreiung der einzelnen Disziplinen mit sich. Die Philosophie erscheint dementsprechend als Versöhuung des logis- 
tischen und positivistischen Prinzips. Die Möglichkeit des Systems beruht darauf, dass die Philosophie sich selbst als einen Teil des seienden Ganzen des Absoluten begreift. Das Absolute ist dann der wahre Einheitspunkt aller Systembildung. „Indem die Philosophie, in deren eigener Wirklichkeit das endlos Mannigfaltige zur Einheit gebracht wird, sich selbst als Teil des von ihr zur Einheit Gebrachten Mannigfaltigen begreift, hat diese Einheit, von welcher die wirkliche Philosophie miterfasst ist, den MannigfaltigkeitenProgressus des Erkennens in sich aufgehoben, ist so darüber hinaus und macht somit das seiende Ganze oder Gott aus". Der Versuch, durch eine Art Synthese beider über Kant und Hegel zugleich hinauszugelangen, ist nicht ohne Beziehung zu jenem, der auf eine Verbindung Kants und Goethes gerichtet ist, und wie letzterer von entscheidender Bedeutung für die Bildung des Philosophierens. In der Schrift Ehrenbergs sehe ich allerdings, sosehr sie auch durch subtile Analysen glänzt, lediglich eine allgemeine Orientierung übèr dieses Programm, von dessen Realisierung nicht Geringes zu erwarten sein dürfte.

Die Unzulänglichkeit einer Philosophie, deren Ergebnisse nicht hinter die wissenschaftliche Theorie zurückgehn, wird namentlich in jenen zahlreichen Schriften betont, die, an der Grenze der strengen Philosophie stehend, die Brücke von dieser zum kulturellen und praktischen Leben suchen. Es hätte keinen Sinn, auf all diese Erscheinungen einzugehn, in denen sich neben ehrlichem Streben und manch tüchtiger Begabung häufig auch ein vager Dilettantismus breitmacht. Insbesondere die Kulturphilosophie hat ja unter Überproduktion und deren Konsequenzen, einer masslosen Wiederholung und Verseichtigung an sich bedeutsamer Motive, zu leiden. In der Forderung einer höheren Form der Erkenntnis als der des abstrahierenden Verstandes, einer Versöhnung von Vernunft und Glaube, Religion und Intellektualismus, stimmen die meisten dieser Schriften überein. Eine populäre Darstellung der Hauptrichtungen der Philosophie bietet Heussner „Die philosophischen Weltanschauungen" (Verlag Vandenhoeck \& Ruprecht, Göttingen, 269 S.). Die Relativität philosophischer Theorien bedarf ihrer Ergänzung in einem religiösen Prinzip, welches der Persönlichkeit allein höchste, widerspruchsfreie Einheit gewähren kann. 
Einen noch ausgesprochener religionsphilosophischen Charakter tragen die Betrachtungen Friedrich von Hindersins (Otto Wigand, Leipzig, 126 S.) eine Verklärung mystischen Weltgefühls.

Einer klaren und gedankenvollen Darstellung des Religionsproblems begegnen wir in Raoul Richters "Religionsphilosophie“, (Ernst Wiegandt, Leipzig, VIII u. $178 \mathrm{~S}$.) die vor allem eine strenge Begriffsbestimmung ihres Gegenstandes sucht. Der Ausgangspunkt hierfür ist eine verallgemeinernde Abstraktion aus den empirischen Religionen, die indessen den wertkritischen Masstabe nicht Genüge leisten kann. Für diesen ist vielmehr erforderlich, dass der empirisch abstrahierte Begriff zu einer absoluten Idee, einer Norm, fortentwickelt werde. Die formalen Bestandteile der Religion werden von den inhaltlichen gesondert. Erstere ergeben sich aus einer. Zergliederung des Verhältnisses zwischen Religion, Kunst und Morạl. Religion ist kein erkennendes, sondern ein wertendes Verhalten; Religionsphilosophie ist dementsprechend die theoretische Beleuchtung, die Erkenutnis dieser Seinsbewertung. Die inhaltliche Charakteristik ergibt die Notwendigkeit einer Welterkenntnis als Grundlage dieser Bewertung. Das Einheitsprinzip der Welt ist Gott, der überpersönliche Allgeist, das Prinzip des Wahren, Guten und Schönen. Die Bewertung führt zur Alternative des Optimismus und Pessimismus, über den hinaus der Verfasser zu einer bedingungsweisen Weltbejahung, nämlich einer Bejahung des als positiv und in diesem Sinne als religiös wertvoll Erkannten strebt. Dem Ideal der Religion kommt in materialer und formaler Hinsicht das Christentum am nächsten und daher bildet es als ideale Kulturgemeinschaft das solideste Fundament für die religiöse Entwicklung der Zukunft.

Vom Zusammenhang des religiösen und philosophischen Lebens handelt auch Maiers Essaysammlung „An der Grenze der Philosophie“ (Tübingen, Mohr, VI u. 405 S.) Melanchthon, Lavater, David Friedrich Strauss werden als Repräsentanten einer zwischen philosophischen und religiösen Interesse geteilten Lebensrichtung vorgeführt. Der problematische Doppelsinn dieser interessanten Physiognomien lässt die Schwächen jener Teilung und einer durch sie bedingten Weltanschauung deutlich zutagetreten. Das Unverlierbare, Positive ist aber andererseits eben darin enthalten, dass hier das Gefübl selber nach logischer Krystallisierung sucht. Insoferne steht diese Schrift in einem engen inneren Zusammenhange mit Maiers bedeutungsvoller Psychologie des emetionalen 
Denkens, die ihrerseits den Urgegensatz des affektiven und logischen Elementes zu überbrücken bestrebt war.

Auch die Erscheinung Friedrich Nietzsches will aus diesem Gegensatze und der Notwendigkeit seiner Überwindung verstanden werden. Dass sie Nietzsche geglïckt ist, kann nicht behauptet werden; zusehr ist sein Wesen zwischen der mystischen Unmittelbarkeit des religiösen Impulses und dem Zwange skeptischer Analyse gespalten. Eben damit aber erweist er sich als ein treuer Repräsentant unserer Zeit und es erklärt sich, dass seine Wirkung auf diese noch immer nicht abgeschlossen ist. Aus der einschlägigen Literatur hebe ich Richard M. Meyers "Nietzsche“ (Beck, München, X u. 702 S.) hervor. Die Schrift ist nicht allein wegen ihres Inhaltes, sondern anch wegen der darin sich entfaltenden Methode von Interesse, welche kein philosophisches, vielmehr ein ausgesprochen literarhistorisches Gepräge trägt. In rveitschichtiger Reproduktion des vorliegenden Materials sucht der Verfasser ans den einzelnen Elementen allmählich Persönlichkeit und Lehre des Philosophen zu konstruieren, während auf eine synthetische Durchdringung, eine plastische Herausarbeitung des Grundtypus Verzicht geleistet wird So stellt sich die Schrift als äusserster Gegenpol von Simmels Goethebuch dar, von dem später zu reden sein wird. Simmel sucht eine einheitliche Formel des Künstlers, Heyer hält' eine solche sogar dem Philosophen gegenüber für ausgeschlossen. Blickt man tiefer, so wird man das Unternehmen Simmels für das berechtigte halten, ja im Sinne einer wirklichen Erkenntnis für das einzig mögliche. Denn es zeigt sich dann, dass auch die literar-historische Methode insgeheim die philosophische voraussetzt. Der Literarhistoriker bedarf ja von Anbeginn einer Direktive zur Auswahl jener Elemente, die ihm das Bild der Persönlichkeit in geeigneter Weise zusammenzusetzen vermögen. Er kann daher das Bestreben des Philosophen, jenes Bild in möglichst eindeutiger, organischer Weise zu entwerfen, nicht tadeln. Eben Meyers Buch ist ein Beweis dafür, dass ein Erschlaffen dieser Tendenz die Gesamtwirkung beeinträchtigen muss. Seine Darstellung verliert sich in die Breite historischen Details und die zweifellos sehr interessanten Züge, die sie häuft, sind nicht imstande, einen einheitlichen Gesamtaspekt von Nietzsches Werk und Weltanschauung zu erwecken. Am wertvollsten scheinen mir hier die Analysen des Zusammenhanges zwischen Nietzsche und der romantischen Kultur. 
Dieseun Gedankenkreise gehört auch eine Schrift von Hermann Franke „Über die Entwicklung der Dinge“ (Berlin, Erust Hofmann, 92 S.) insoferne an, als sie den Nachweis versucht, dass der Weltprozess irgendein Mal alle Möglichkeiten durchlaufen muss und sonach einer ewigen Wiederkunft des Gleichen Raum gibt.

Die idealistische Tendenz der modernen Philosophie bekundet sich zumal in ihrer Anwendung auf Kultur und Leben als ein entschiedener Protest gegen den einseitigen Prozess theoretischer und praktischer Mechanisierung. Von diesem Geiste ist auch Walther Rathenaus "Zur Kritik der Zeit" getragen (Berlin, S. Fischer, 260 S.) Der Verfasser geht in der Charakteristik des modernen Menschen nicht bis zu den letzten Abstraktionen zurück wie Ehrenberg in seiner "Geschichte des Menschen unserer Zeit* (im Alpha-Omega Verlag in Heidelberg.). Umso plastischer aber ist das Bild der Zeit, das er entrollt. Geschichte wird aus der Schichtung zweier ungleichartiger. völkischer Elemente erklärt. Der Mechanisierungsprozess der Techuik wird in etwas gewaltsamer Weise lediglich aus der Übervölkerung abgeleitet, obne dass es an idealen Perspelrtiven mangelt, die eiue Überwindung dieses Zustandes herbeiführen könuen.

So energisch die Nletaphysik nunmelur auch in der deutschen Philosophie einzusetzen beginnt, eines lässt sie der reinen Erkenntuistheorie gegenüber ziveifellos im Nachteile erscheinen: dass letztere ein geschlossenes Ganzes wesentlich gloichgerichteter Bestrebungen repräsentiert, während die metaphysischen Tendenzen einen solch geneinsamen Brennpunkt noch nicht gefunden baben. Sie vorlaufen zur Zeit in unbestimmten Linieu, deren Ziele eher geahnt, als klar bezeichnet werden können! Der wissenșchaftliche Realismus, dessen wirksamste Darstellung wir Külpe verdanken, wird schwerlich die grosse Anfgabe der Philosophie erfüllen, ein einheitliches Weltbild zu schaffeu; zu wenig löst er die alstrakten Grundbegriffe der Wissenschaften in letzte Elemente des unmittelbaren Seins auf. Soweit bisher grosszügige Versuche in dieser Richtung unternommen wurden, scheinen sie an Stelle der alten Weltanschaunngen, die sich um die zentrale Idee des Geistes oder des Willens gruppieren, eine Philosophie des Lebens treten zu lassen. Hier hatte Bergsons "Schöpferische Entwicklung." die in deutscher Übertragung erschienen ist (Eugen Diederichs, Jeua, 371 S.) 'wegweisend gewirkt. Bergson versucht, nachdem er den Dualismus von Materie und Geist aufs Äusserste gespannt hat, inn wiederum 
in dem einheitlichen Prinzip des Lebens aufzulösen. Das Leben wird ihm dabei zur metaphysischen Potenz, die sich in zwei Richtungen, einer aufsteigenden und einer herabsinkenden entfaltet; einer, die zur energischen Konzentration des Ich führt, und einer, die in Materialität und Räumlichkeit erstarrt. Dieser metaphysischen Potenz werden wir weder durch Wahrnehmung noch durch abstrakte Begriffe inne, wohl aber durch intuitire Einsenkung in die Unmittelbarkeit des Lebensprozesses, dessen Wesen „konkrete Daner" ist. Darin stimmt Bergson mit dem Pragmatismus überein, dass ihm das Logische einen lediglich vitalen, praktischen Wert besitzt. Zum Unterschiede vom Pragmatismus aber erblickt er darin nicht den Weg zur Wahrheit, den bloss die Intuition $\mathrm{zu}$ bereiten vermag. Unverkeunbar trägt Bergsons Lebensidee Merkmale an sich, die weder der deskriptiven Biologie, noch der deskriptiven Psychologie entnommen sind, da sie Wertungen enthalten. Als solche aber sind sie mit der Relativierung des Logischen unverträglich, sondern fordern eine tiefere Durchdringung desselbeu mit den emotionalen Motiv der Intuition. Es fehlt auch der-deutschen Literatur nicht an Werken, denen der Plan eines ähnlichen Universalisnus der Lebensidee vorschwebt. Hatte schon Hermaun Keyserling in seinen "Prolegomena zur Naturphilosophie" solch ein Unternehmen vor Augen gehabt, so hat Karl Joël in seinem Werke "Seele und Welt" (Eugen Diederichs, Jena, VII u. 424 S.) diesem Gedanken ein weit kräftigeres Fundament gegeben. Seine Schrift, die in der Form vielleicht weniger glücklich ist, als im Inhalte, soll die Einseitigkeiten des Materialismus und des Idealismus überwinden, dadurch dass sie die Dualität des Seelischen und Körperlichen anerkennt, sie abcr gleichwohl aus einer höheren Einheit, einem Indifferenzpunkte zu entwickeln sucht. Deutlich sind hier die Nachwirkungen Schellings, deutfich die Beziehungen zu Bergson. Nicht umsonst nennt Joël sein Werk den Versuch einer organischen Auffassung. Auch ihm ist die Materie erstarrtes Leben. Ein und dasselbe Urprinzip entfaltet sich in materiellen und im psychischen Sein. Dort zerfällt es in eine beziehungslose Mannigfaltigkeit, hier fasst es sich zu einer höchsten Einheit zusammen. Auf den Neuhegelianismus weist die Überwindung abstrakter Gegensätze durch das konkrete Leben der Idee hin. Das"Absolute ist daher auch weder im pantheistischen noch im theistischen Sinne zu verstehn; weder dadurch, dass Gott durch die Welt, noch dass die Welt durch 
Gott aufgesogen wird. Es besteht hier im Grunde dasselbe Verhältnis wie zwischen Materie und Geist. So erweist sich Joël darin, Bergson gegenüber, als Vertreter der deutschen Philosophie, dass er den Trieb zur höchsten Synthese und Einheit nicht unterhalb des Logischen, sondern in diesem selber vorgezeichnet sieht.

Kritische Bedenken gegen den Plan einer Lebensphilosophie äussert Rickert in seinem Logos-Aufsatze „Lebenswerte und Kulturwerte". Er wendet sich vor allem mit Recht gegen eine biologische Fundierung der Philosophie. Abgesehen davon, dass gegen eine solche dieselben Einwände in Kraft treten, die gegen den Psychologismus erhoben wurden: die Unmöglichkeit, das Ganze der Welt aus einem Teilausschnitt derselben, einem speziellen Forschungsgebiete, zu erklären, wird hier auf den irrefïhrenden Doppelsinn des Teleologischen hingewiesen. Telos bedentet ebensowohl Zweck als auch Ende Endergebnis, Resultat. Weil die Biologie teleologisch verfährt, könnte es scheinen, als ob sie ihre Betrachtungen unter den Gesichtspunkt des Z auf Werte hinweisen, so scheint es weiter, als ob auf diese Betrachtungen eine wertende Weltanschaunng begründet werden könnte. In Wirklichkeit aber verfügt die Biologie über keine Wertmasstäbe, positive oder negative, und sie hat daher auch kein Recht, von Zwecken zu sprechen. Es fehlt ihr ja das wertsetzende Moment, der Wille. Sie spricht bloss von Endergebnissen, zu deren Herbeischaffung bestimmte organische Phänomene als. Mittel und Bedingungen dienen. Die teleologische Auffassung enthüllt sich hier demnach im Grunde ats eine konditionale. Dass anch die Öbertragung ins Psychische den Lebensbegriff in keinerlei unnittelbare Beziehung zum Kulturbegriff, zur Idee des kulturellen, philosophischen Wertes setzt, sucht Rickert in den weiteren Ausführungen zu beweisen. Der Grundgedanke von dem diese geleitet werden, ist der: überall, wo wertbetonte Gebilde entstehen, muss die Unmittelbarkeit des Lebens schon irgendwie überwunden sein. Nicht allein in der Philosophie, sondern auch in der Kunst und im praktischen Gestalten. Zumal in der Sphäre logischer Werte ist es völlig evident, dass sie gerade durch ein vollkommenes Abrücken aus jener Sphäre erst zur Entstehung gelangen können. Die Argumente Rickerts sind den mannigfachen Spielarten des Biologismus gegenüber völlig im Rechte. Es hat sich uns gezeigt; dass auch seine höchsten Repräsentanten, wie Nietzsche und Bergson, diesen Einwänden zum Teile preisgegeben sind. Nicht 
minder die Lebensphilosophie des französischen Denkers Guyan, der, ein Zeitgenosse Nietzsches, zu diesem maucherlei Beziehungen hat. Der philosophisch-soziologischen Bücherei gebührt das Verdienst, einen Teil seiner Schriften in deutscher Übersetzung veröffentlicht zu haben. Ich erwähne hier ,.Die Kunst als soziologisches Phänomen “ (Leipzig, Verlag von Dr. Ḱlinkhardt, IV. u. 503 S.) und "Die ästhetischen Probleme der Gegenwart" (Leipzig, Klinkhardt XII. u. 230 S.). Eine zusammenfassunde Darstellung gibt Ernst Bergmann in der Monographie "Die Philosophie Guyaus" (Leipzig, Klinkhardt, 144 S.). Vije Nietzsche und Bergson foiert Guyau in Leben die schöpferische Weltmacht. Alle Abstraktion, alle Starrhejt des Begriffes erscheint schon ihm, diesem Vorläufer des Intuitivismus, als eine Terfälschnng der in ewiger Bewegung sich entfaltenden Wirklichkeit. Aus ihrem Borne müssen Ethik, Religion und Kunst scböpfeu. Dementsprechend verwirft er ebensowohl den normativen Charakter der Ethik und ihre Zurückführung auf die Instanz des Gewissens, wie die dogmatische und konfessionelle Einschränkung des religiösen Gefühls, wie die feststehenden Typen nnd Idealbilder der Kunst. Alle sollen vielmehr im Strom unmittelbaren Lebens aufgelöst werden, dessen Charakter Guyau in altruistischer Hingabe und Ferschwendung, in unaufhaltsamer Mitteilung und Terbindung erblickt; - zum Unterschiede von Nietzsche, für den Leben zwar nicht der Ausdruck des Egoismus, wohl aber jener höheren Form der Exklusivität und Abschliessung ist, die durch den Machtwillen bezeichnet wird. Schon hier zeigt es sich, dass der Begriff des Lebcus ein vieldentiger ist, dass er bei weitem nicht jene logische Klarheit besitzt, die ihm ohne kritische Durchlenchtung die Eignung verleihen könute, das Fundament einer philosophischen Weltanschanung zu bilden. Zweifellos haben die genaunten Denker, Guyau, Nietzche, Bergson, eine ganz bestimnte .Ethik und Logik schon als Ausgangspunkt; und ihre Auffassung des Lebens ist bereits die Anwendung und Konsequenz dieses Ausgangspunktes. In den erwähnten Deutungen erschöpft sich aber nicht der Sinn des Lebensbegriffes. Wenigstens scheint mir Goldscheids Lebenstheorie, wie sie in seinem Werke „Höherentwicklnng und Menschenökonomie" (Philosophisch-soziologische Bücherei, Leipzig, Klinkhardt, XXVI. u. 664 S.) zum Ansdruck kummt, unter keines der geuannten Schemen zu fallen. Weit eher schliesst sie sich an jene Gedankengänge an, die von Avenarius und Mach und einem Teil des moderuen Pragmatismus, den 
sogenannten Denkökonomen, vertreten werden. Nicht Selektion noch schrankenlose Verschwendung ist demnach die Forderung des Lebens, vielmehr Sparsamkeit. Blos durch die Erfüllung dieser Forderung scheint eine Höherzüchtung der Menschheit möglich. Alle bestehenden Elemente müssen aufs intensirste ausgenützt und in den Dienst gemeinsamer Zwecke gestellt werden. So tritt die soziale Ethik den Lebenstatsachen nicht mehr als eine fremde Norm gegenüber: sie britigt deren innerste Notwendigkeit zum Ausdruck. Dieser Auffassung entspricht es ferner, dass Goldscheid ein entschiedener Fürsprecher der mechanistischen Naturphilosophie ist, die er zumal gegen den Neovitalismus und verwandte Strömungen verteidigt. An seine Stelle babe der Neomechanismus zu treten, der allen Kräften eine bestimmte Richtung zuweist. Mier drängt sich freilich der Gegeneinwand auf, dass zwischen den Richtungen physikalischer, chemischer und biologischer Kräfte eine zu erhebliche Abweichung besteht, um die Subsumtion unter eine einzige Gruppe zu erlauben.

Diese Vielfältigkieit des Lebensphänomens, die sich in der Pluralität seiner logischer Bestimmungen spiegelt, beweist die Berechtigung der von Rickert geübten Kritik. Auch die ist von grösster Bedeutung: dass der Lebensprozess in seiner elementaren Unmittelbarkeit etwas Irrationales ist, aus dem niemals die Erklärung rationaler Setzungen, wie sie in allem gegenständlichen Schaffen verkörpert sind, entnommen werden kann. Gleichwohl scheint mir eine Vertiefung der Lebensidee in dem Sinne möglich, dass sie nicht mehr jenseits der Logizität und Rationalität verharrt, sundern mit dieser zu einer umfassenden universalen Einheit verknüpft wird. Eine solche Idee kann freilich niemals aus deskriptive Biologie oder deskriptiver Psychologie abstrahiert werden. Hier tritt das logistische Argument in Kraft, dass aus blossen 'Patsachen niemals Werte zu dẹduzieren sind. Es gibt aber eine Sphäre der Transzendentalpsychologie, in der die Sphären des Seins und des Geltens, der Tatsachen und der Werte, zwar nicht zur Deckung gelangen, aber immerhin auf einen gemeinsamen Schnittpunkt weisen. Ihn' haben die nachkantischen Identitäts. philosophen zum Ausgangspunkte freilich allzu phantastischer Weltkonstruktionen genommen. Und in dieser Region ist der Lebensidee auch ein tieferer Sinn abzugewinnen.' Sie offenbart sich hier als Synthese des Irrationalen und des Rationalen, der Bewegung und der Gestalt. Im Ich hat der Lebensprozess in seinem un- 
aufhörlichen zeitlichen Ablauf zugleich ein ruhendes Prinzip der Gestaltung und Organisation, was schon Kant, der hier, gleichsam voransschauend, dem radikalen Evolutionismus Bergsons die Spitze abbog, in seiner Lehre von der transzendentalen Synthese der Apperzeption, fixiert bat. Es ist indessen möglich, dass sich auch im äusserlichen Lebensprozesse der körperlichen Welt Analogien solch einer Synthese des Irrationalen und Rationalen, des dynamischen Werdens und des gestalteten Seins, der Bewegung und des Formprinzips, nachweisen lassen. Dann wäre es möglich, ohne sich an den Postulaten des Logismus und der Wertlehre zu versündigen, die Idee des Lebens zu einer für das philosophische IVeltbegreifen zentralen zu erheben.

Auch an solchen Versuchen fehlt es nicht. Eben der "Logos", der ja, wie schon sein Name sagt, dem Geiste des strengen logischen Transzendentalismus entstammt, wird vielleicht einer im kritischen Sinne unternommenen Verbindung von Erkenntnislehre und Metaphysik die Wege bereiten. Hier hebe ich eine Reihe ron Aufsätzen hervor, die als Anzeichen einer solchen Bewegung betrachtet werden können. Einer genaueren Lektüre derselben bleibt es nicht verborgen, dass sowohl bei den Repräsentanten des logischen wie bei denen des metaphysischen Philosophierens sich das unabweisbare Bedürfnis nach gegenseitiger Ergänzung hervordrärigt. Das beweist nicht minder Hermann Keyserlings Aufsatz "Über das Wesen der Intuition und ihre Rolle in der Philosophie“, als Friedrich Steppuhns. "Die Tragödie des mystischen Bewusstseins:". Aber auch Richard Kroners gehaltrolle Arbeit "Zur Kritik des philosophischen Monismus" und Mehlis' Essays "Die platonische Liebe" und "Formen der Mystik" müssen in diesem Zusammenhange angeführt werden. Richard Kroner isoliert zunächst in tiefdringender Analyse die beiden Grundrichtungen des Philosophierens, die analytische und die synthetische, und gelangt zu dem Ergebnisse, dass in der analytischen Philosophie selber durch eine notwendige Erkenntnis ibrer inneren Schrankeu die synthetische zu ihrem Rechte gelangen muss. Die analytische Philosophie reisst die kategoriale Denkform und den denkfremden Inhalt auseinander; die synthetische will beide Gegensätze ineinander auflösen, indem sie den Widerspruch selbst zur Wahrheit stempelt. Der absolute Monismus ist aber uumöglich; blos das Äquivalent eines solchen ist dadurch gegeben, dass die analytische Philosophie die syuthetische als ein berechtiges Moment 
in sich aufnimmt. „Bloss zum Selbstberwusstsein und zur Selbstbestimmung ibrer Grenzen muss die analytische Philosophie erweckt werden. Nicht ausserhalb des Denkens oder vor ihm darf sie ein Unbestimmtes, Fremdes behaupten. . Sie muss einsehen, dass es vielmehr in ihm selbst liegt, in dem Widerspruche, den das Denken in 'sich selbst erzeugt. Indem die Logik der Philosophie den durch die Ansprüche des synthetischen Denkens entstandenen Widerspruch als das denkfremde Element und zugleich als das notwendige Resultat des analytischen Denkens erkennt, entdeckt sie im Felde der Analyse selbst die Forderung der Synthese, bringt sie den zur Widerspruchslosigkeit hinstrebenden monistischen Trieb als einen legitimen auch dem analytischen Denker innewohnenden Trieb zum Bewusstsein und macht die Vorstellung einer Gegensätzlichkeit beider Denkarten im Sinne der Ausschliessung der einen durch die andere zunichte". So wird die analytische Philosophie in bestimmtem Sinne danach streben müssen, synthetisch zu werden. Dies Streben wird aber den Charakter einer idealen Annäherung haben, das heisst: im endlichen, beschränkten Denken wird das Unendliche als ein Sollen wirksam sein. So löst sich zuletzt der Grundgegensatz beider Richtungen in den des Seins und Sollens auf.

In seiner schönen Studie "Die platonische Liebe" findet Mehils zugleich Gelegenheit aus dem Wesen des Eros den tieferen Sinn dieser Philosophie zu entwickeln. "Wir dürfen Plato nicht. zum Mystiker machen, der die grosse Negation am Endlichen vollzieht, noch dürfen wir inn als Freund des sinnlichen Daseins denken, der in sinnlicher Schönheitssehnsucht das Übersinnliche vergisst; sondern er gebört zu jenem Zwischenreich der grossen dämonischen Naturen, der wahrhaft erotischen Menschen, die der Geringfügigkeit und Alltäglichkeit des Endlichen den grossen Zug und den leidenschaftlichen Aufschwing zu einem höheren Dasein geben." Die Formen der Mystík untersucht Mehlis in dem gleichnamigen Aufsatz. Die Schwierigkeit ist es auch hier, das Irratiouale zu rationalisieren, dem Begriffslosen begriffliche Prägung zu verleilren. Mystisches Erleben kann zunächst auf zweifache Weise geformt werden: durch den Begrif und durch das Symbol. Nach dem Ziele der Mystik muss man unterscheiden das Ideal der Gotteserkenntnis, der Gottesminne und das mehr ins Praktische gewendete eines vollkommenen Lebens. Im Anschlusse an Windelband charakterisiert Mehlis zwei Typen des Mystikers, den passiven, 
dessen Wesen Hingabe, Auflösung im Allsein ist; den aktiven, dessen Wesen Steigerung des individuellen Willens zu universaler Schöpfungskraft ist, wie ihm der Magier repräsentiert. Beide Richtungen zu vereinigen strebt Meister Ekkehard, der aber in der Unerfüllbarkeit dieses Strebens zugleich den tragischen Grundkonflikt alles mystischen Weltempfindens erlebt.

Die innere Verbindung des Mythos und des Logos, in der wir die Aufgabe der zukünftigen Philosophie erkannten, scheint mir näher gerückt in einer Reihe von Werken, die erkenntnistheoretische Analyse mit dem Versuch wetaphysischer Synthese verknüpfen. Hier nenne ich vor allem Hans Driesch, der von der Biologie ausging, dann aber die Unzulänglichkeit ejnes an einem speziellen Wissensgebiet orientierten Standpunktes erfassend, die Notwendigkeit empfand, zu den Grundformen des Deukens zurückzugehen und in einer allgemeinen Logik und Erkenntnislehre den umfassenden Plan einer Weltanschauung zu ziehen.

In seiner grossangelegten "Philosophie des Organischen" hatte Driesch sich um Erneuerung des Vitalismus in einem durchaus kritischen, von Phantastik freien Sinne bemüht und das Ergebnis seiner in empirischer wie in logischer Hinsicht exakten Ausführungen ist die Autonomie des Lebens, seine Unauflösbarkeit in mechanische Vorgänge. Er hält sich von jeder Verquickung des Lebensproblems mit Elementen eines unklaren Panpsychismus fern. Der Zusammenhang des körperlichen und geistigen Lebens, auf den es der metaphysischen Weltdeutung vor allem ankommt, ist nicht durch vage Analogien, sondern bloss durch ein System festgefügter Begriffe zu konstruiereu. Diesem Zwecke soll Driesch' neues Werk „Ordnungslehre“, ein System des nichtmetaphysischen Teiles der Philosophie (Diederichs, Jena, S. 355) entsprechen. Die Ordnungslehre ist lediglich die Vorbereitung zur eigentlichen Philosophie des Erkennens: der Metaphysik. Sie hat, ohne noch die Frage nach der Wirklichkeit $\mathbf{z n}$ stellen, das Inventar des Geistes nach seinen immanenten Beziehungen zu durchsuchen und zumal den inneren Aufbau dieser Beziehungeu darzustellen. Von den Ursetzungen Sein, Dasein, Sosein, Anderssein, und ihren Spezifizierungen geht der Verfasser zur Ordnung des Naturwirklichen und der Ichsphäre über, um zum Schlusse Ausblicke auf Erkenntnislebre und Metaphysik zu eröffnen. Man kann diesen Plan der Ordnungslehre als einen Impressionismus des Denkens bezeichnen. Nicht als ob das Denken in Impressionen aufgelöst 
würde. Eben dies widerspricht ja der streng logistischen Tendenz des Verfassers; aber es wird das Denken noch gar nicht in seinem realistischen Gehalt, sondern lediglich in seiner logischen nicht etwa psychologischen Erscheinungsform gewürdigt. Die rationalen Ordnungen sollen sich in der Weise entfalten, dass sie auch mit den extremsten Voraussetzungen des Solipsismus vereinbar wären. Bestimmte Begriffe treten mit dem Anspruche auf Realisierung auf; sie sind anf ein Wirkliches gerichtet, als ob innen ein Wirkliches entspräche, wobei dies "als ob" vom Standpunkte der Ordnungslehre als Symbol und nicht - wie bei Vaihinger - als Fiktion aufzufassen ist. Auch auf die Psychologie wird diese Betrachtungsweise ausgedehnt. Die Realisierungen haben hier, wie in der Aussenwelt, zunächst bloss als Ordnungstypen zu erscheinen. So wird Erinnerung als ein bewusstes Haben von irgendetwas definiert, das mit dem Zeichen des Vergangenes-Bedeutens versehen ist.

Die Ordnungslehre entspricht dem, was sonst, freilich nicht wach Driesch' Terminologie, als reine Erkenntnistheorie bezeichnet wirl. Jene daseinsfreie Betrachtung der Kategorien, die auch Meinongs gegenstandstheoretische Untersuchungen bezwecken. Dass eine solche Perspektive möglich, sogar notwendig ist, kaun nicht bezweifelt werden. Jedenfalls aber ist die von ibr aus aufgenommene Welt ein starres Abstraktionsprodukt, eine Summe blinder Zeichen, Bedeutungen und Schemen, die jeglicher sinnvollen Erfüllung ermangeln. Gerade dieser extreme Logismus, der das Denken der Werte völlig von dem der Realitäten sondert, verrät in seinen letzten Konsequenzen, dass dort, wo nicht die Überzeugung von einer Wirklichkeit entsteht, anch der Wert sich auflösen muss. Driesch fasst sein Unternehmen daher auch bloss als Vorstufe der Metaphysik Erst die Vollendung dieser wird uns ein erschöpfendes Urteil tiber die Bedeutung des ganzen Werkes ermöglichen. Denn zwieifellos hat er sich eine Aufgabe gestellt, von der die Weiterentwicklung der Philosophie abhängt: die Verbindung der Lehre vom richtigen Denken mit der vom wabren Erkennen und vom wirklichen Sein. Fü: eine Pbilosophie des Lebens, wie für jede Metaphysik überhaupt, ist vor allem eine Auseinandersetzung mit Logik und Erkenntnistbeorie geboten. Die Einseitigkeiten der Bergson'schen Lehre liegen ja hauptsächlich darin, dass sie die Intuition zu schroff und unversöhnlich dem Prinzip der Rationalität entgegensetzt; dass sie somit den 
Logos durch den Mythos verdrängen lässt: während die Philosophie der Zukunft eine Synthese beider wird suchen müssen.

Das Streben nach einer solchen Synthese wird auch in Georg Simmels neuesten Werken sichtbar. Schon in seiner „Philosophischen Kultur" und insbesondere in seinem Goethe-Buch. Die "Philosophische Kultur" (Leipzig, Verlag von Klinkhardt, 319 S.) enthält eine Sammlung von Essays, die, soweit sie auch dem Thema nach auseinanderliegen, gleichwohl durch zweierlei Momente verknüpft sind, die sich, näher geschaut, freilich in ein einziges auflösen: die Methode und das unsichtbare ideelle Leitmotiv. Die Methode wird von Simmel selbst in der Einleitung charakterisiert: indem die philosophischen Behauptungen unvereinbar weit auseinanderliegen und keine unbestrittene Geltung besitzen: indem dennoch etwas Gemeinsames in ihnen gespürt wird, dessen Wert alle Anfechtung des einzelnen überlebt nnd den philosophischen Prozess weiter und weiter trägt, kann jenes Gemeinsame nicht in irgendeinem Inhalte, sondern bloss in diesem Prozess selber liegen." Das unsichtbare Leitmotiv ist die Idee des vielgestaltigen Lebens. Und indem auch der Denkprozess sich diesem anzuschmiegen, die. Plastik seines Materials sich anzueignen sucht, wird er selber zum Lebensprozess. So ist es auch für Simmel charakteristisch, dass Form und Inhalt, Methode und Gegenstand im letzten Grunde bei inm zusammenfallen. Wir finden hier Studien psychologischer, aesthetischer, kulturphilosophischer und religionsphilosophischer Natur. Das allmähliche Werden dessen, was wir als letzte Gemeinsamkeit der verschiedenen Liebensfunktionen herausspüren, soll hier aus der Form der subjektiven Impression in die des objektiven Begriffes gehoben werden; und zwar fasst Simmel den Charakter des Lebens hier als einen tragischen. Es ist seinem tiefsten Wesen nach auf ein Absolutes gerichtet und bleibt dennoch in die Relativitat von Beziehungen und Gegensätzen gebanut. Schon in dem ersten Aufsatze, einer Analyse des Abenteuers, tritt dieser tragische Widerstreit des Relativen und Absoluten hervor. Noch intensiver beherrscht er das Geschlechtsproblem: Die Differenzierung des Seins in ein männliches und weibliches ist Ausdruck für die allem Lebenden eingeborene Notwiendigkeit, zur Setzung und Erfassung seiner selbst erst durch die Beziehung 'auf ein anderes zu gelangen. Aber der menschliche Geist will nicht in dieser Relativität gefangen bleiben: die vom Manne aufgestellten Werte beanspruchen 
absolute Gültigkeit. Auch im Kulturproblem, in Ästhetischen und Religiösen offenbart sich diese Tragik, die namentlich an der Gestalt Michelangelos zu packender Darstellung gebracht wird.

Noch enger ist die Beziehung des "Goethe" (Verlag Klinkhardt \& Biermann, Leipzig, VII n. 264 S.) zur Metaphysik des Lebèns. Weit entfernt von jeder bloss literarhistorischen Methode, fasst Simmel Goethe als Reprüsentauten einer bestimmten universalen Lebensrichtung; er fragt nach dem geistigen Sinn der Goetheschen Existenz uiberhaupt. Das Wichtigste ist die Untersuchung uiber Goethes Auffassung der Wahrheit. Diese bestimmt er an den Werte, den eine Vorstellung nicht ihrem za objektivierenden Inbalte nach, sondern als Funktion, als Lebenselement für die Totalität des Lebens gewinnt. Darin ist auch der Unterschied vou der pragmatistischen Erkenntnislehre begründet. Für den Pragmatismus ist es der Inhalt der Vorstellung, dessen Förderlichkeit ihr den Wahrheitswert gibt, „für Goethe ist es der Prozess ihres Vorstellens, die lebendige Funktion, die sie im $\mathrm{Zn}$ sammenhang der seelischen Entwicklung übt." Eine solche Auffassung ist freilich bloss innerbalb eines Weltbildes möglich, dem das Erkeunen des Einzelnen als ein Teil des universalen Welterlebens, dem das Subjekt mithin als ein Stück des absoluten Seins erscheint. Dies ist der grosse Gegensatz zwischen dem Universalismus Goethes und dem Dualismus Kants, dem der Übergang vom Sein zum Erkennen eine prinzipielle Variation bedeutet. Es ist, im tiefsten Grunde geschaut, wiederum der Gegensatz des Mythischen und des Logischen, den wir überall dort finden, wo Intuition und Intellekt einander gegenüberstehn. Die Vereinigung beider bezeichneten wir schon als Aufgabe der Zuknnft. Gelingt es, die Synthese Goethes mit Kants Analyse zu vereinigen, dann werden nicht bloss einer neuen grossen Weltauschauung, sondern einer neuen geistigen Kultur überhaupt die Wege geebnet sein. 